\title{
Impact of Ferromagnetic Layer Thickness on the Spin Pumping in Co60Fe20B20/Ta Bilayer Thin Films
}

\section{Soumyarup Hait}

Indian Institute of Technology Delhi

\section{Sajid Husain}

Universite Paris-Saclay

Nanhe Kumar Gupta

Indian Institute of Technology Delhi

Nilamani Behera

University of Gothenburg

Ankit Kumar

Uppsala university

Rahul Gupta

Uppsala university

Vineet Barwal

Indian Institute of Technology Delhi

Lalit Pandey

Indian Institute of Technology Delhi

Peter Svedlindh

Uppsala university

Sujeet Chaudhary ( $\sim$ sujeetc@physics.iitd.ac.in )

Indian Institutie of Technology Delhi https://orcid.org/0000-0002-0535-6132

\section{Research Article}

Keywords: Ferromagnetic resonance, Spin pumping, Damping, Co60F20B20, heavy metal, Spin current

Posted Date: March 11th, 2021

DOI: https://doi.org/10.21203/rs.3.rs-277397/v1

License: (c) (1) This work is licensed under a Creative Commons Attribution 4.0 International License.

Read Full License 
Version of Record: A version of this preprint was published at Journal of Materials Science: Materials in Electronics on April 17th, 2021. See the published version at https://doi.org/10.1007/s10854-021-058769. 


\title{
Impact of ferromagnetic layer thickness on the spin pumping in $\mathrm{Co}_{60} \mathrm{Fe}_{20} \mathrm{~B}_{20} / \mathrm{Ta}$ bilayer thin films
}

\author{
Soumyarup Hait ${ }^{\mathrm{a}, 1}$, Sajid Husain ${ }^{\mathrm{b}, 1}$, Nanhe Kumar Gupta ${ }^{\mathrm{a}}$, Nilamani Behera ${ }^{\mathrm{c}}$, Ankit Kumar ${ }^{\mathrm{d}}$, \\ Rahul Gupta ${ }^{\mathrm{d}}$, Vineet Barwal ${ }^{\mathrm{a}}$, Lalit Pandey ${ }^{\mathrm{a}}$, Peter Svedlindh ${ }^{\mathrm{d}}$ and Sujeet Chaudhary ${ }^{\mathrm{a},{ }^{*}}$ \\ ${ }^{a}$ Thin Film Laboratory, Indian Institute of Technology Delhi, New Delhi 110016, India \\ ${ }^{b}$ Unité Mixte de Physique, CNRS, Thales, Université Paris-Saclay, 91767 Palaiseau, France \\ ${ }^{c}$ Department of Physics, University of Gothenburg, 41296 Gothenburg, Sweden \\ ${ }^{d}$ Department of Materials Science and Engineering, Uppsala University, Box 35, SE-751 03 Uppsala, Sweden \\ ${ }^{1}$ Authors contributed equally \\ Corresponding author: sujeetc@iitd.ac.in
}

\section{Research Highlight:}

1. Growth of three different series of $\mathrm{Co}_{60} \mathrm{Fe}_{20} \mathrm{~B}_{20}(\mathrm{CFB}, 4,6$ \& 8nm) with $\mathrm{Ta}(1-10 \mathrm{~nm}$ in step of $1 \mathrm{~nm}$ ) using ion-beam sputtering technique.

2. Structural and morphological study using X-ray diffraction (XRD), X-ray reflectivity (XRR), Atomic Force Microscopy (AFM) and Energy Dispersive X-ray (EDX) analysis.

3. A detailed analysis of spin pumping based on range of $\mathrm{Co}_{60} \mathrm{Fe}_{20} \mathrm{~B}_{20}$ and $\mathrm{Ta}$ thicknesses.

4. Low thicknesses of $\mathrm{Co}_{60} \mathrm{Fe}_{20} \mathrm{~B}_{20}(4 \& 6 \mathrm{~nm})$ follow normal spin pumping behaviour while anomaly is present in thick $\mathrm{Co}_{60} \mathrm{Fe}_{20} \mathrm{~B}_{20}(8 \mathrm{~nm})$ film heterostructure.

\begin{abstract}
:
We report the tuneable spin angular momentum transfer (spin pumping) from $\mathrm{Co}_{60} \mathrm{Fe}_{20} \mathrm{~B}_{20}$ (CFB) amorphous alloy into the Ta heavy metal nanolayers. All the films are grown on Si (100) substrate at room temperature using ion-beam sputtering technique. Structural studies reveal that the grown Ta films over amorphous CFB are crystalline even at ultrathin regime. The bilayers possess very low interface roughness $(<0.5 \mathrm{~nm})$ and are continuous throughout the thickness range. Comparative analysis of the spin pumping in CFB (4, 6 and $8 \mathrm{~nm})$ as a function of the Ta thickness (vary from 1 to $10 \mathrm{~nm}$ in step of $1 \mathrm{~nm}$ ) has been performed employing ferromagnetic resonance (FMR) spectroscopy. It is observed that the effective
\end{abstract}


damping increase exponentially with the increase of Ta i.e., follows ballistic spin transport) in two series of $\mathrm{CFB}(4 \mathrm{~nm}) / \mathrm{Ta}(0-10 \mathrm{~nm})$ and $\mathrm{CFB}(6 \mathrm{~nm}) / \mathrm{Ta}(0-10 \mathrm{~nm})$ bilayers, which is characteristic of normal spin pumping. However, the anomalous behaviour has been observed for CFB $(8 \mathrm{~nm}) / \mathrm{Ta}(0-10 \mathrm{~nm})$ bilayer series where the spin current generated in Ta with the thicker CFB behaves oppositely. The results demonstrate the strong dependence of ferromagnet thickness on the spin pumping into the Ta nanolayers. This study paves the way to choose suitable ferromagnetic layer thickness for spin current induced switching applications in spintronics.

\section{keywords}

Ferromagnetic resonance; Spin pumping; Damping; $\mathrm{Co}_{60} \mathrm{~F}_{20} \mathrm{~B}_{20}$; heavy metal; Spin current

\section{Introduction}

Spin transport through the interface of ferromagnetic (FM) and nonmagnetic (NM) materials with high spin orbit coupling (SOC) have gained a severe interest in the last decade because of the spin-orbit torque based low energy MRAM applications. Spin pumping is one of the efficient methods of producing pure spin current where due to magnetization precession in FM, pure spin current transfers to adjacent NM [1]. The spin transport or more precisely, spin angular momentum transport from FM to NM through the FM-NM interface leads to an evolution in the damping constant of the FM materials as the spin of FM relaxed in the adjacent NM layer. Ferromagnetic resonance (FMR) is one of the renowned methods to find this damping evolution and hence to study this spin relaxation. Tserkovnyak et al. have provided the insightful information about interfacial spin relaxation mechanism in FM/HM bilayer system using FMR [2].

A bilayer composed of a FM and a heavy metal (HM) layer with large SOC is a promising system for studying such damping evolution. There are several HMs that are explored via spin pumping and among these the most studied HMs are the $4 \mathrm{~d}$ and $5 \mathrm{~d}$ transition metals, such as Pt [3,4], Pd [5,6], $\beta-\mathrm{W}$ [7-9], $\beta-$ Ta [10-12], and Mo [13,14], etc. It should be noted that the ' $d$ ' orbital occupancy of these HMs plays a major role in their performance [15]. The spin angular momentum transfer from FM to HM critically depends on the interface quality. It is understandable that the HM and FM layers in different thicknesses can affect the electronic structure of each other close to the interface and hence modify the interactions between the 
layers. On changing the thickness of FM layer, the interfacial SOC changes and can produce additional Rashba spin splitting which modifies the spin current transport through the interface [16-20]. Paul Haney et al. theoretically reported that the spin current generation from a FM to HM majorly depends on the two phenomena; one is the interface effect, which deals with interfacial SOC that gives Rashba interactions and second due to bulk SOC of HMs [21]. They have proposed a semi-classical approach to understand these two phenomena together using a Boltzmann formalism and shown the effect of changes in FM and HM layer thickness on the spin dynamics. However, the detailed experimental study on the effect of FM layer thickness on spin pumping in FM-HM bilayer is still lacking.

In this work, the detailed analysis of spin pumping in $\mathrm{Co}_{60} \mathrm{Fe}_{20} \mathrm{~B}_{20}$ (CFB)/Ta bilayer heterostructure is demonstrated using FMR technique. In these bilayers, the CFB thicknesses were fixed as 4, 6, and $8 \mathrm{~nm}$ for three different series and Ta thickness was varied from 1 to 10 $\mathrm{nm}$ in the step of $1 \mathrm{~nm}$ for all CFB thicknesses. This particular bilayer structure is a very promising structure for MRAM application as CFB possesses excellent magnetic properties in terms of texture, spin polarization, damping constant etc [22][8]. Also the $\mathrm{Ta} / \mathrm{CFB} / \mathrm{MgO} / \mathrm{CFB}$ based Magnetic Tunnel Junction (MTJ) shows very promising results for MRAM application [1][23][24]. So, it will also be very crucial to study the spin dynamic properties of this particular interface. In-plane FMR measurements were performed to study the spin pumping phenomena on all the samples at room temperature.

\section{Experimental details}

A series of CFB thin films of thicknesses $4,6,8,10,15$, and $20 \mathrm{~nm}$ were grown on naturally oxidized Si (100) substrate at room temperature using an ion-beam sputtering deposition system (NORDIKO-3450). The details about the deposition system can be found in Ref. [25]. For film deposition, the 6 inch alloy target (atomic composition Co60\% Fe20\% B20\%), fixed on a remote-controlled water-cooled turret, was sputtered by high energy Ar ion beam $(500 \mathrm{eV})$ extracted from a RF ion source of $\sim 4.5 \mathrm{in}$. diameter. The ion grid current/voltage parameters were fixed at $\mathrm{V}+=500 \mathrm{~V}, \mathrm{I}+=79 \mathrm{~mA}, \mathrm{~V}-=-270 \mathrm{~V}$, and $\mathrm{I}-=5 \mathrm{~mA}$. All the CFB films were deposited at working pressure of $\sim 8.4 \times 10^{-5}$ Torr at RF power of $100 \mathrm{~W}$ at room temperature. Further, three series of CFB with 4, 6, and 8nm thickness and Ta films on the top with thickness $\mathrm{t}_{\mathrm{Ta}}$ ranging from 1 to $10 \mathrm{~nm}$ in step of $1 \mathrm{~nm}$ were deposited in-situ at room temperature. The growth of CFB thin films and their magnetic properties were reported in Ref. 
[26]. The Ta thin films are grown at $3.4 \times 10^{-4}$ Torr (at RF power of $95 \mathrm{~W}$ ) with base vacuum of $\sim 1 \times 10^{-7}$ Torr. For deposition of Ta thin films, the grid parameters were changed to $\mathrm{V}+=700 \mathrm{~V}$, $\mathrm{I}+=140 \mathrm{~mA}, \mathrm{~V}-=-270 \mathrm{~V}$, and $\mathrm{I}-=10 \mathrm{~mA}$. For sputtering, Ar gas was directly fed at $4 \mathrm{sccm}$ flow rate into the RF-ion source.

The structural analysis was performed using X-ray diffraction (XRD) and X-ray reflectivity (XRR) technique using a Panalytical X-ray diffractometer where $\mathrm{x}$-ray of $\mathrm{Cu} \mathrm{k} \alpha$ was used. The surface morphology of the films was investigated using Atomic force microscopy (AFM) technique (Bruker, model no: dimension ICON). The elemental analysis of the samples were carried out via energy dispersive X-ray spectroscopy technique (Hitachi High tech.).The CFB/Ta bilayer films were cut into $1 \mathrm{~mm} \times 4 \mathrm{~mm}$ to record the broadband FMR spectra employing a home-built FMR set-up [10]. The microwave signal was sourced from Vector Network Analyzer (HP make Model-8719 ES) for a range of frequencies from 4-10 $\mathrm{GHz}$ to the co-planar waveguide (CPW) placed in between the electromagnet. The data was collected in DC-magnetic field sweep mode by keeping the microwave frequency fixed. To improve the signal to noise ratio, the DC magnetic field was modulated using an AC field of an optimized strength of $0.13 \mathrm{mT}$ at $211.5 \mathrm{~Hz}$ frequency, which was obtained by powering a pair of Helmholtz coils from the reference oscillator of the lock-in amplifier from Stanford Research Systems Inc. (Model-SR 830 DSP). The output signal, essentially the derivative of the signal from the sample, locked at $211.5 \mathrm{~Hz}$ was detected by the lock-in amplifier via an RF-diode. All the measurements were performed at room temperature.

\section{Results and Discussion}

\section{A. X-Ray Diffraction (XRD) Study:}

Glancing incidence XRD (GIXRD) measurements (with an incident angle of $1^{\circ}$ ) of CFB films with different thickness was performed and the spectra are shown in Figure (1). The spectra show a common presence of a broad peak near $44.5^{\circ}$, which suggests the highly nanocrystalline (or amorphous) growth of CFB films[27][28]. Fitting this hump using combination of a Gaussian and a Lorentzian function, the full width at half maxima is calculated, which is in the range of $8^{\circ}-12^{\circ}$, yielding a crystallite size less than $1 \mathrm{~nm}$. Thus, from XRD, the amorphous nature of CFB is established in these sample series. 


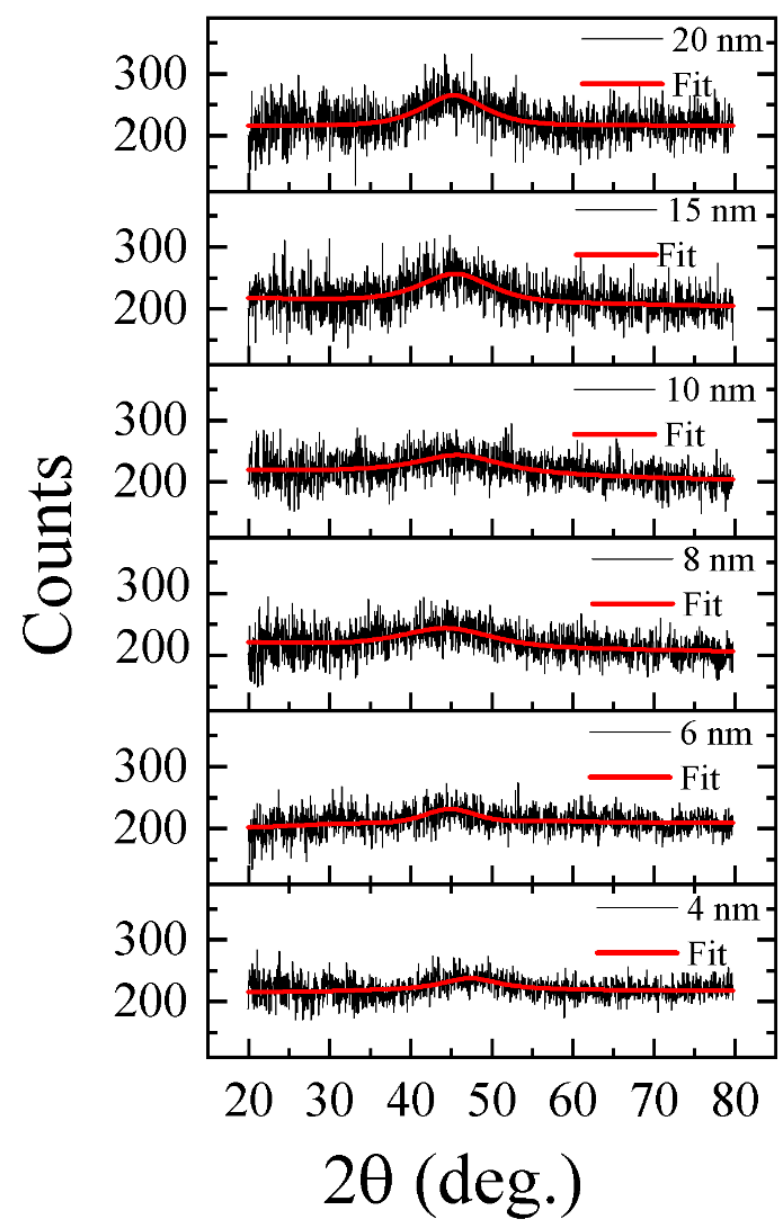

Figure (1): GIXRD spectra of CFB films of different indicated thicknesses $\mathrm{t}_{\mathrm{CFB}}$ in $\mathrm{nm}$.

To find out the phase of the grown Ta films over CFB, GIXRD measurements (with an incident angle of $1^{\circ}$ ) were performed on bilayer samples with different Ta thickness. Figure (2) shows XRD spectra taken for different thickness of Ta grown on $4 \mathrm{~nm}$ amorphous CFB. The presence of (002) diffraction peak near $\theta=33.5^{\circ}$ and (011) peak near $38.5^{\circ}$ conclusively suggests the formation of mixed phases, i.e., $\alpha-T a$ and $\beta$-Ta in these samples. However, it is reported that the phase of Ta thin films can be tuned by tunning the sputtering rate, energy of the sputtered species and working pressure [29][30]. The presence of (011) peak of $\alpha$-Ta of even $3 \mathrm{~nm}$ nominally thin Ta film in the bilayer conclusively suggests that the Ta films grew crystalline over amorphous CFB even at ultrathin regime unlike previous report [31]. It should be noted that the nominal value of Ta-film thicknesses (e.g., $\mathrm{t}_{\mathrm{Ta}}=3,4,6, \& 10 \mathrm{~nm}$ ) also include $\sim 2 \mathrm{~nm}$ thin $\mathrm{Ta}_{2} \mathrm{O}_{5}$ in all cases, as revealed by XRR analysis (c.f. Table-I). 


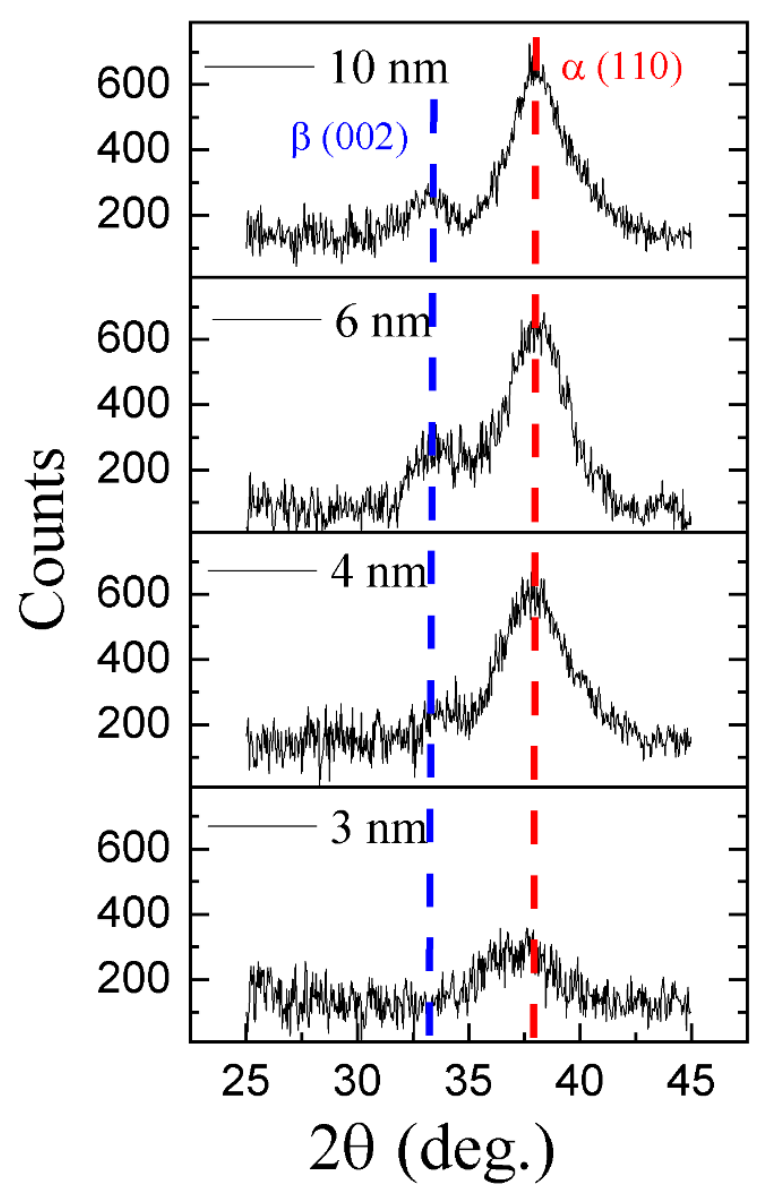

Figure (2): GIXRD spectra of CFB (4 nm)/ Ta $\left(\mathrm{t}_{\mathrm{Ta}}=10,6,4,3 \mathrm{~nm}\right)$ bilayers. The bleu (red) dash lines mark the $2 \theta$ positions of the most intense peaks of the $\beta$-Ta $(\alpha-\mathrm{Ta})$ phase as per JCPDS standards.

\section{B. X-ray reflectivity (XRR) Study:}

X-ray reflectivity spectra were recorded on these bilayer samples to find out their exact thickness, density and interface roughness. The clear Kiessig fringes over the whole measurement range suggest the impressive quality of the films and their interface. The recorded spectra are fitted with X-ray reflectivity software (segmented V.1.2) considering a stack of $\mathrm{Si} / \mathrm{SiO}_{2} / \mathrm{CFB} / \mathrm{Ta} / \mathrm{Ta}_{2} \mathrm{O}_{5}$. The thickness of each layer matches with the assumed (or nominal) value quite well and the low interface roughness of the films suggests that there is no intermixing between $\mathrm{CFB}$ and Ta in our films. Also, we have previously reported the cross-sectional measurement where no interface mixing was observed [32]. It should be 
noted that interface roughness has a very crucial impact on spin pumping and also ferromagnetic coupling. A rough interface between a FM-NM results in degradation in spin transport channels as it impacts the SOC of the interface as well as the spin impedance matching [33][12]. However, the high-quality interface (evident from the XRR results) in our samples, rule out the roughness effect in our samples. The simulated values of the thickness, density and interface roughness of each layer of few samples are presented in Table-1. The credit behind such a good quality interface is goes to the unique growth process of NORDIKO 3450 ion beam sputtering system, where the plasma formed in a separate chamber which results in very low working pressure of the range of $10^{-5}$ Torr which is two orders of magnitude lower than that in normal magnetron sputtering process. Also, in the sputtered species travel with minimal collisions leading prominently a line-of-site deposition such that very little/negligible shadowing effect occurs. 


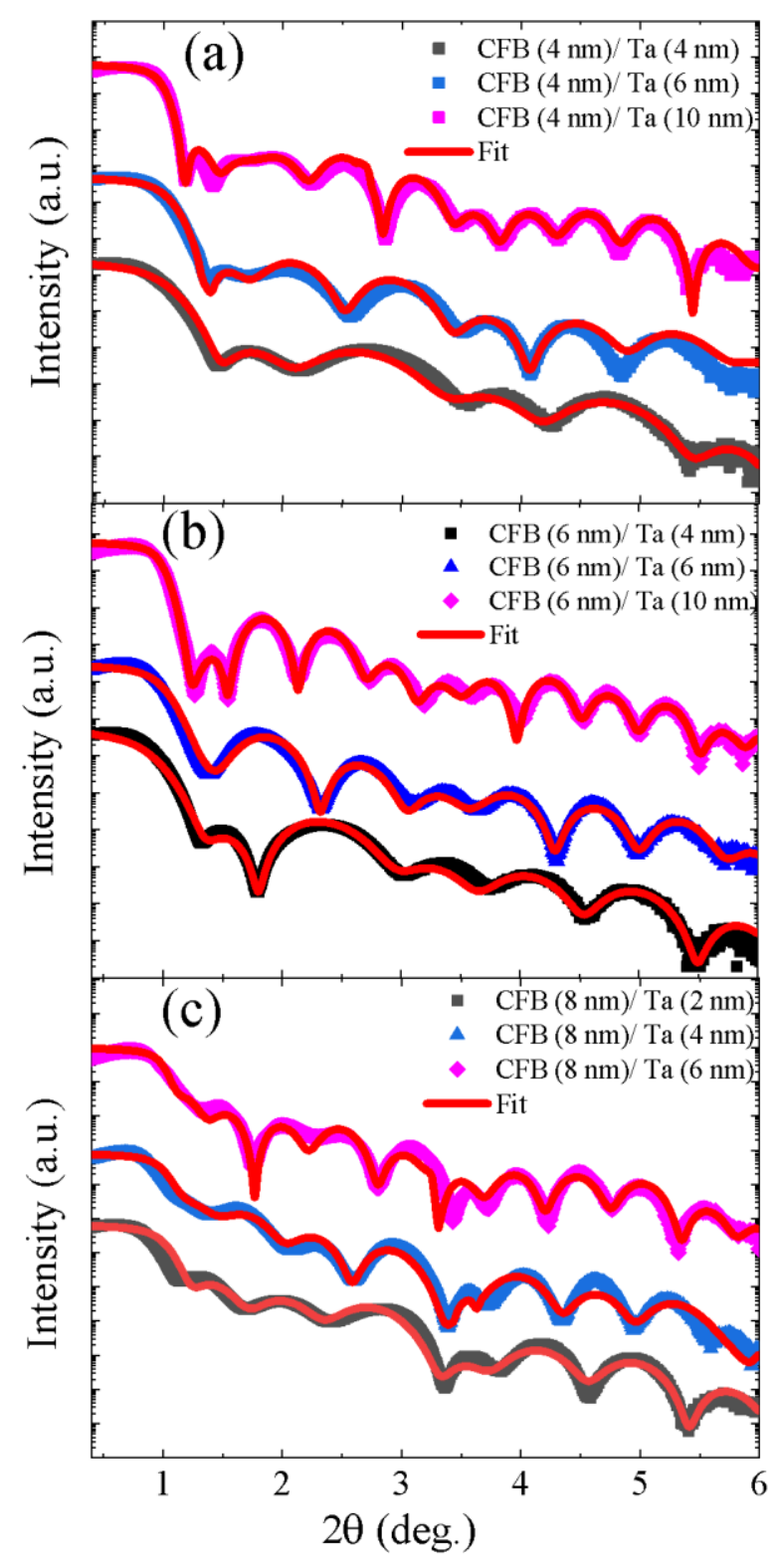

Figure (3): XRR spectra of (a) CFB (4 nm)/ Ta (4, 6 , $10 \mathrm{~nm}),(\mathrm{b}) \mathrm{CFB}(6 \mathrm{~nm}) / \mathrm{Ta}(4,6,10 \mathrm{~nm})$ and (c) CFB $(8 \mathrm{~nm}) / \mathrm{Ta}(4,6,8 \mathrm{~nm})$. Red lines represent the fitted spectra.

Table 1: The stimulated values of density, thickness and roughness of each layer of few samples are shown. In the sample name column, the number in the parenthesis is film thickness expressed in $\mathrm{nm}$. 


\begin{tabular}{|c|c|c|c|c|c|c|c|c|c|}
\hline \multirow{2}{*}{ ample } & \multicolumn{3}{|c|}{ Density $\left(\mathrm{gm} / \mathrm{cm}^{3}\right)$} & \multicolumn{3}{|c|}{ Thickness (nm) } & \multicolumn{3}{|c|}{ Roughness (nm) } \\
\hline & FD & & $\mathrm{Ta}_{2} \mathrm{O}_{5}$ & CFB & & & & & (2) \\
\hline $\mathrm{CFB}(4) / \mathrm{Ta}(4)$ & $\begin{array}{r}9.8 \\
\pm 0.8\end{array}$ & & $\begin{array}{c}9.01 \\
\pm 0.24\end{array}$ & $\begin{array}{c}3.85 \\
\pm 0.31\end{array}$ & $\begin{array}{r}1 . \\
\pm 0\end{array}$ & & & & \\
\hline $\mathrm{Cl}$ & & & & & & & & & \\
\hline $\mathrm{CFB}(4) / \mathrm{Ta}(10)$ & & & & & & & & & \\
\hline $\mathrm{CFB}$ & & & & $\begin{array}{c}5.59 \\
\pm 0.07\end{array}$ & & & & & \\
\hline $\mathrm{CFE}$ & $\begin{array}{c}7.74 \\
\pm 0.74\end{array}$ & & & $\begin{array}{c}5.78 \\
\pm 0.63\end{array}$ & & & $\begin{array}{c}0.39 \\
\pm 0.23\end{array}$ & & \\
\hline $\mathrm{CFB}$ & $\begin{array}{c}6.3 \\
\pm 0.82\end{array}$ & & & $\begin{array}{c}5.79 \\
\pm 0.96\end{array}$ & & & $\begin{array}{c}0.47 \\
\pm 0.13\end{array}$ & & \\
\hline CFB & $\begin{array}{c}8.33 \\
\pm 0.39\end{array}$ & 16 & $\begin{array}{r}8 . \\
\pm 0\end{array}$ & $\begin{array}{c}7.63 \\
\pm 0.89\end{array}$ & $\begin{array}{r}1 . \\
\pm 0\end{array}$ & $\begin{array}{r}2 . \\
\pm 0\end{array}$ & $\begin{array}{c}0.41 \\
\pm 0.17\end{array}$ & $\begin{array}{c}0.26 \\
\pm 0.04\end{array}$ & $\begin{array}{c}0.32 \\
\pm 0.13\end{array}$ \\
\hline $\mathrm{CFB}($ & $\begin{array}{c}9.29 \\
\pm 0.81\end{array}$ & $\begin{array}{l}15 . \\
\pm 0 .\end{array}$ & $\begin{array}{r}8 . \\
\pm 0\end{array}$ & $\begin{array}{c}7.77 \\
\pm 0.84\end{array}$ & $\begin{array}{r}4 . \\
\pm 0 .\end{array}$ & & $\begin{array}{c}0.37 \\
\pm 0.16\end{array}$ & & \\
\hline $\mathrm{CFB}$ & $\begin{array}{c}9.22 \\
\pm 0.94\end{array}$ & $\begin{array}{c}15 \\
\pm 0.65\end{array}$ & $\begin{array}{c}8.71 \\
\pm 0.14\end{array}$ & $\begin{array}{c}7.24 \\
\pm 0.25\end{array}$ & $\begin{array}{c}6.07 \\
\pm 0.21\end{array}$ & $\begin{array}{c}1.98 \\
\pm 0.14\end{array}$ & $\begin{array}{c}0.31 \\
\pm 0.13\end{array}$ & $\begin{array}{c}0.17 \\
\pm 0.09\end{array}$ & $\begin{array}{c}0.26 \\
\pm 0.12\end{array}$ \\
\hline
\end{tabular}

\section{Atomic Force Microscopy (AFM) Study:}

Figure (4) shows the AFM topography of the various single layer CFB films of different thicknesses. The surface of these films is found to be very flat with roughness below $\sim 0.5 \mathrm{~nm}$, which could also be limited by the instrument tip. However, from the AFM images, it is also reconfirmed that there is no sign of large grain formation in the CFB layers as also seen in XRD results (discussed above). 

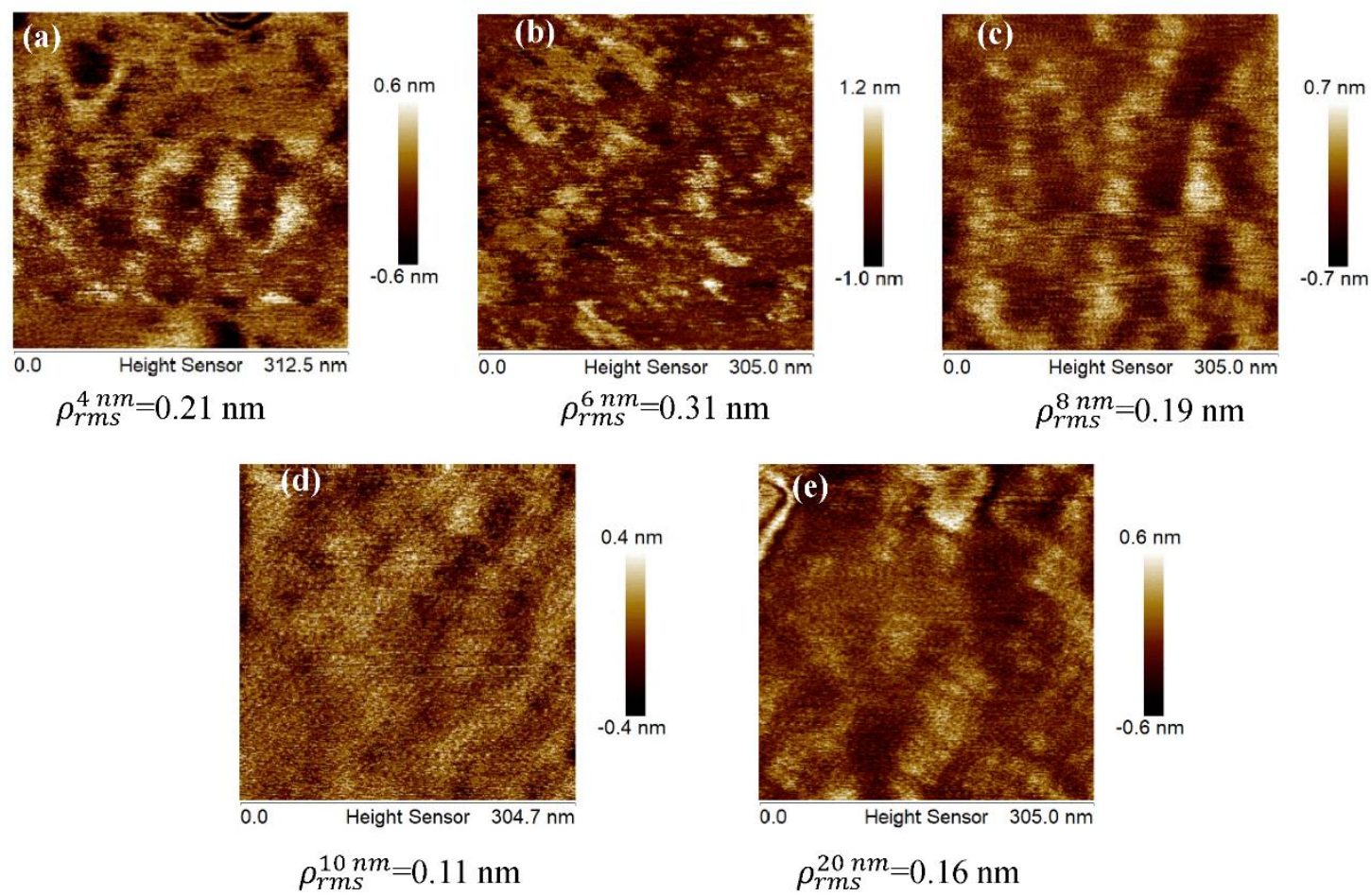

Figure (4): Topography of single layer CFB thin film of different thickness, (a) $4 \mathrm{~nm}$, (b) 6 $\mathrm{nm}$, (c) $8 \mathrm{~nm}$, (d) $10 \mathrm{~nm}$, and (e) $20 \mathrm{~nm}$.

\section{Elemental Analysis:}

Energy dispersive X-ray spectroscopy was performed on various Ta capped CFB thin films. All the samples shows that the CFB films are stoichiometric with the atomic ratio of Co and Fe is $\sim 3$. These measurements were performed over a large scan area of $7.6 \times 7.6 \mu \mathrm{m}^{2}$ with electron beam of energy $15 \mathrm{keV}$ to elucidate the continuous feature of the films. The EDX mapping is shown in Figure (5) which confirms the continuous growth of the CFB and Ta films with thickness lower than $5 \mathrm{~nm}$. The credit of such continuous growth even at this lower thickness of film is also goes to our unique ion beam sputtering system as discussed above. 

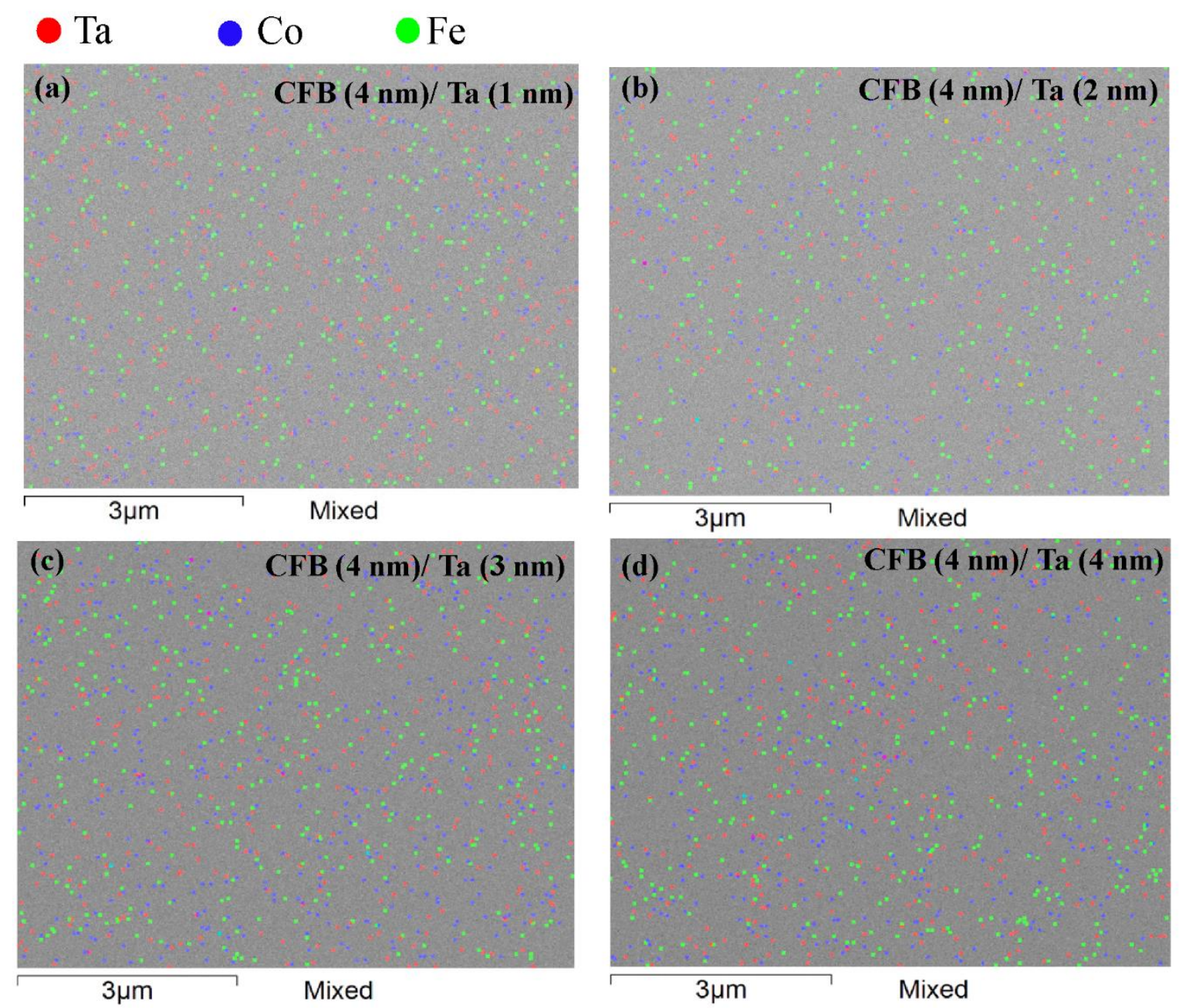

Figure (5): EDX mapping of (a) CFB (4 nm)/Ta (1 nm), (b) CFB (4 nm)/Ta (2 nm), (c) CFB $(4 \mathrm{~nm}) / \mathrm{Ta}(3 \mathrm{~nm})$ and (d) CFB $(4 \mathrm{~nm}) / \mathrm{Ta}(4 \mathrm{~nm})$, respectively. The red, blue and green points represent $\mathrm{Ta}$, $\mathrm{Co}$ and $\mathrm{Fe}$ respectively. These images clearly reveal the composition homogeneity in these samples.

\section{E. Ferromagnetic Resonance Analysis}

Figure 6(a) shows the FMR spectra recorded on the CFB single layer $(8 \mathrm{~nm})$ thin film. The FMR spectra were fitted using the voltage derivative $\mathrm{dV} / \mathrm{dH}$ of symmetric and anti-symmetric Lorentzian functions to extract the line-shape parameters such as resonance field $\mu_{0} H_{r}$ and linewidth $\mu_{0} \Delta H$, given by [34], 


$$
F M R \text { signal }=S \frac{\partial \frac{\Delta H^{2}}{\left\{\Delta H^{2}+\left(H_{\text {ext }}-H_{r}\right)^{2}\right\}}}{\partial\left(H_{\text {ext }}\right)}+A \frac{\partial \frac{\Delta H\left(H_{\text {ext }}-H_{r}\right)}{\left\{\Delta H^{2}+\left(H_{\text {ext }}-H_{r}\right)^{2}\right\}}}{\partial\left(H_{\text {ext }}\right)}
$$

where $\mathrm{S}$ and $\mathrm{A}$ are the coefficients corresponding to symmetric and anti-symmetric Lorentzian functions, respectively. $\mu_{0} \Delta H$ is full-width at half maximum (FWHM) linewidth of FMR spectrum, $\mu_{0} H_{r}$ is the resonance field of microwave absorption and $\mu_{0} H$ is the in-plane applied DC magnetic field.
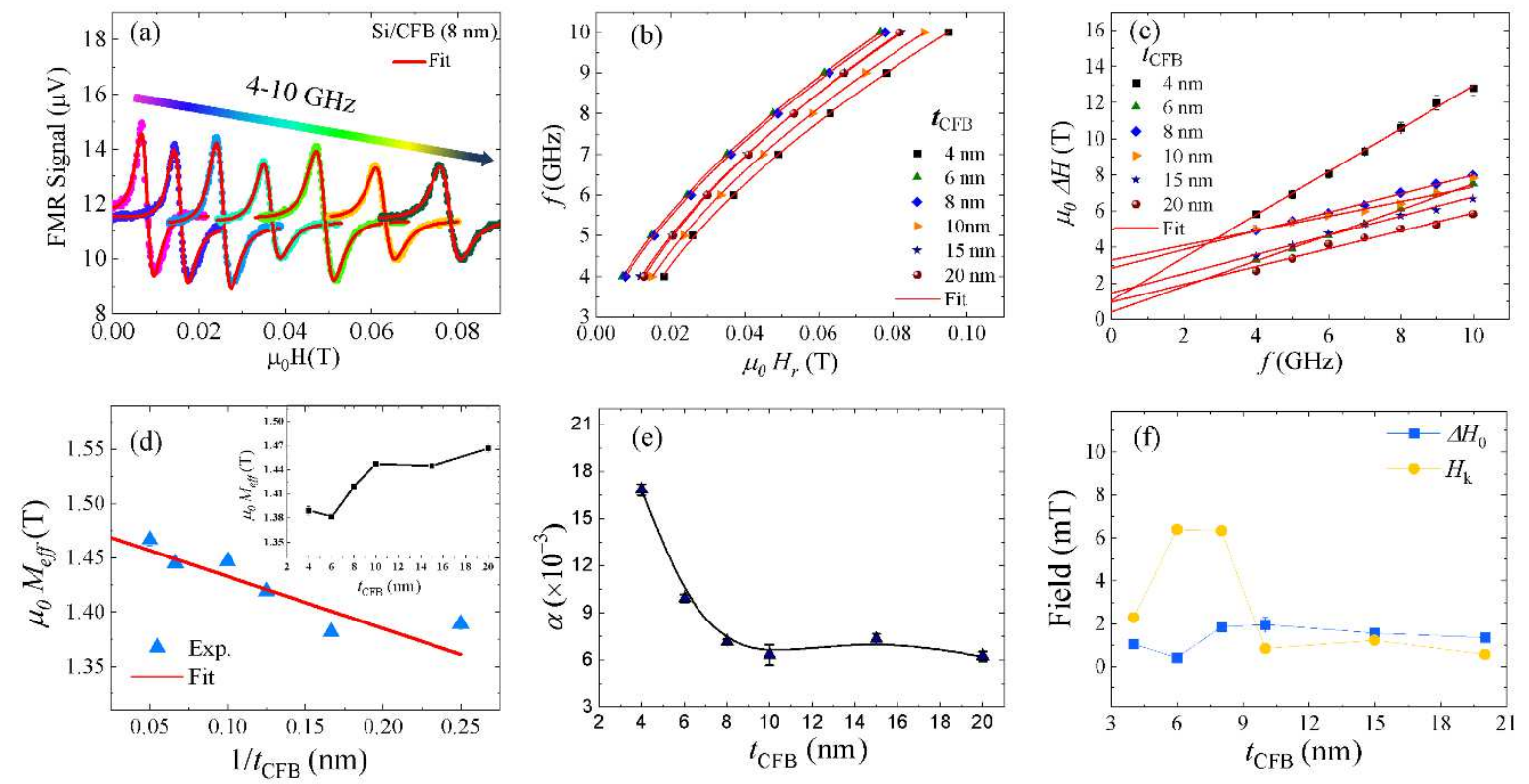

Figure 6: (a) FMR spectra of $8 \mathrm{~nm}$ single layer CFB thin film. Filled circles are the experimental data points and red lines shows the fitting using Eq. (1). (b and c) Variation in resonance field and linewidth, respectively, as a function of microwave frequency. (d, e and f) Calculated effective magnetization, anisotropy field and inhomogeneous linewidth of the films as a function of thickness of CFB layer.

Figure 6(b) shows the extracted $\mu_{0} H_{r}$ vs. $f$ data for a series of single layer CFB thin films. These curves were fitted using the Kittel's formula [35],

$$
f=\frac{\gamma}{2 \pi} \sqrt{\left(H_{r}+H_{k}\right)\left(H_{r}+H_{k}+4 \pi M_{e f f}\right)}
$$


Where, $\gamma=\frac{g \mu_{B}}{\hbar}$ is the gyromagnetic ratio, $g$ is the Lande's spectroscopic splitting factor and $\hbar$ is the reduced Plank's constant, $\mu_{0} M_{e f f}$ is the effective magnetization, and $\mu_{0} H_{k}$ is the anisotropy field. Inset of Fig. 6(d) shows the calculated $\mu_{0} M_{\text {eff }}$ for all CFB single layer thin films. The values of $\mu_{0} M_{e f f}$ are found to increase with the increase in the CFB thickness $t_{C F B}$ and saturate at large thickness. The variation of effective magnetization is observed in thinner films is possibly due to the surface anisotropic field, which is defined as, $\mu_{0} M_{\text {eff }}=\mu_{0} M_{s}+\mu_{0} H_{S}$, where $\mu_{0} M_{S}$ is the saturation magnetization and $\mu_{0} H_{S}=\frac{2 K_{S}}{\mu_{0} M_{S} t_{C F B}}$ is the surface anisotropy field. Here, $K_{S}$ is the surface anisotropy energy density. Now, to evaluate surface anisotropy energy, the $\mu_{0} M_{\text {eff }}$ vs. $1 / t_{\text {CoFeB }}$ data (Fig. 6(d)) is fitted using the relation obtained by combining the above two relations, i.e.,

$$
\mu_{0} M_{e f f}=\mu_{0} M_{S}+\frac{2 K_{S}}{\mu_{0} M_{S} t_{C F B}}
$$

From the linear fitting using Eq. 3, the value of $\mu_{0} M_{s}$ is found to be $1.48 \pm 0.02 \mathrm{~T}$, which is comparable to the measured value of saturation magnetization $(\sim 1.5 \mathrm{~T})$ [26]. The surface anisotropy energy $K_{S}$ was found to be $-0.028 \pm 0.007 \mathrm{~J} / \mathrm{m}^{2}$, where the negative sign of the anisotropy indicates the magnetization lies in the film plane. Further, the anisotropy fields $\mu_{0} H_{k}$ in these CFB single layers are calculated using Eq. (2) and shown in Fig. 6(f) along with $\mu_{0} \Delta H_{0}$ (discussed later). It is clear that the finite contribution of the anisotropy in these films, which increases on lowering the $t_{C F B}$ results in the low effective magnetization in thinner films.

Now, the Gilbert damping constant $\alpha$ values for the CFB single layer thin film series have been calculated using the linewidth relation,

$$
\mu_{0} \Delta H=\mu_{0} \Delta H_{0}+\frac{4 \pi \alpha f}{\gamma}
$$

Here, $\mu_{0} \Delta H_{0}$ is the frequency independent contribution from magnetic field inhomogeneity effect, while the second term corresponds to the frequency dependent contribution associated with the intrinsic Gilbert damping constant $\alpha$. For bilayers, this $\alpha$ becomes, $\alpha_{\text {eff }}=\alpha_{S P}+\alpha_{C F B}$ , named as effective damping constant, which includes the intrinsic Gilbert damping of single layer CFB $\left(\alpha_{C F B}\right)$ and a spin pumping contribution $\alpha_{S P}$ from the CFB to $\beta$-Ta heavy metallic layer (discussed in the forthcoming section). Figure 6(c) shows the $\mu_{0} \Delta H \mathrm{vs}$. $f$ data for CFB thin films for various thicknesses and fitted with the Eq. (4). The calculated values of Gilbert 
damping for all the single layer CFB thin films are shown in Fig. 1(e). The values of $\alpha$ is found to be enhanced in CFB (4nm), which is expected due to the enhanced in surface scattering contribution at lower CFB thickness [36][37]. It is seen that the values of $\alpha$ do not change beyond the $8 \mathrm{~nm}$ thickness of CFB thin film grown over Si substrates. The saturated value of $\alpha$ matched with reported bulk value of CFB [32]. On the basis of these results, we have chosen three CFB thickness, i.e., 4, 6, and 8nm for making the 3 bilayers series using the non-magnetic HM Ta layer on the top of CFB with HM thickness varying from 1 to $10 \mathrm{~nm}$ in step of $1 \mathrm{~nm}$, to study the effect of FM thickness on the spin pumping in FM/HM bilayers.

\section{F. Spin Pumping in CFB/Ta Heterostructures}

Figure 7 shows the FMR spectra recorded on CFB (4, 6 and $8 \mathrm{~nm}) / \mathrm{Ta}(4 \mathrm{~nm})$ (for brevity we are showing FMR spectra only for $\mathrm{t}_{\mathrm{Ta}}=4 \mathrm{~nm}$ ) bilayer thin films series. By fitting of these FMR spectra with Eq. (1), line-shape parameters (resonance field $\mu_{0} H_{r}$ and linewidth $\mu_{0} \Delta H$ ) are extracted.
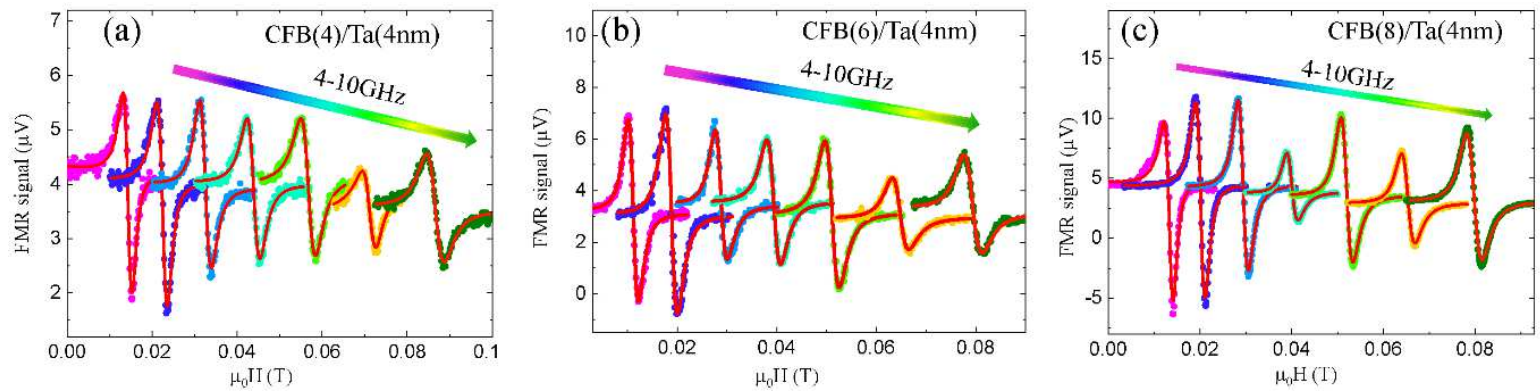

Figure 7: FMR spectra recorded on (a) $\mathrm{CFB}(4 \mathrm{~nm}) / \mathrm{Ta}(4 \mathrm{~nm})$, (b) $\mathrm{CFB}(6 \mathrm{~nm}) / \mathrm{Ta}(4 \mathrm{~nm})$ and (c) $\mathrm{CFB}(8 \mathrm{~nm}) / \mathrm{Ta}(4 \mathrm{~nm})$ bilayer thin films. Filled circles are the experimental data points and red lines shows the fitting using Eq. (1).

The evaluated $\mu_{0} H_{r}$ vs. $f$ are shown in Fig. 4(a-c) for all the three bilayer sample series. Layer stacks are mentioned on top of the corresponding figures. The values of effective magnetization and the anisotropy field of all the samples have been extracted by fitting the $\mu_{0} H_{r}$ vs. $f$ plots using the Kittel Eq. (2). The calculated values of effective magnetization are shown in Fig. 8(df). It can be clearly seen that the $\mu_{0} M_{e f f}$ is increasing as a whole towards the bulk value (the dotted lines) when the thickness of CFB is increased to $6 \mathrm{~nm}$ and $8 \mathrm{~nm}$. Further, the anisotropy $\mu_{0} H_{k}$ calculated from fitting are shown in Fig. 8(g-i). It can be seen that the $\mu_{0} H_{k}$ values are very small for a series of CFB (4)/Ta (1-10). However, the $\mu_{0} H_{k}$ values as a whole are larger in 
thicker CFB (6 \& 8)/ Ta (1-10) series. This indicates that there are finite changes in the magnetic properties of a series with thick CFB. However, the effective magnetization does not show significant variation in these two CFB $(6 \& 8 \mathrm{~nm})$ bilayer series.

CFB(4nm)/Ta(0-10nm)
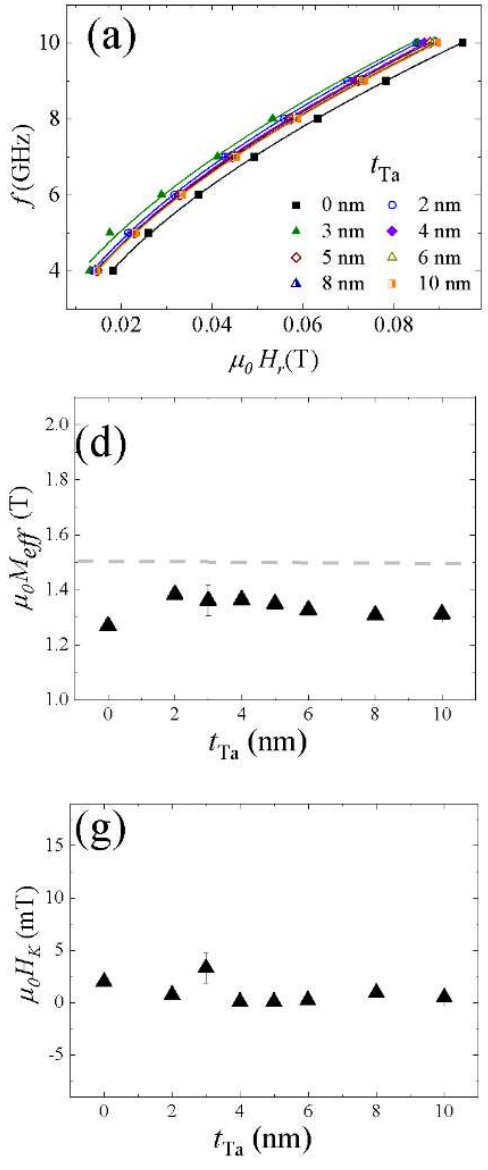

CFB(6nm)/Ta(0-10 $\mathrm{nm})$
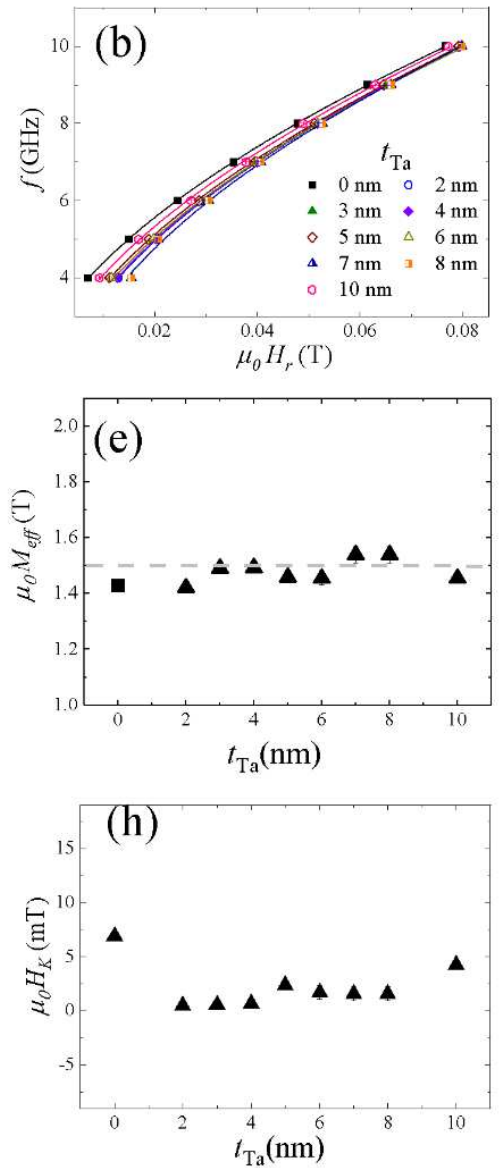

CFB(8nm) $/ \mathrm{Ta}(0-10 \mathrm{~nm})$

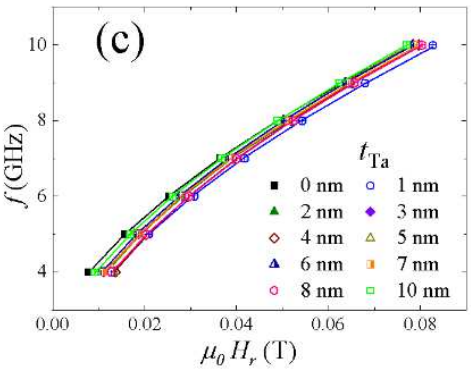

(f)

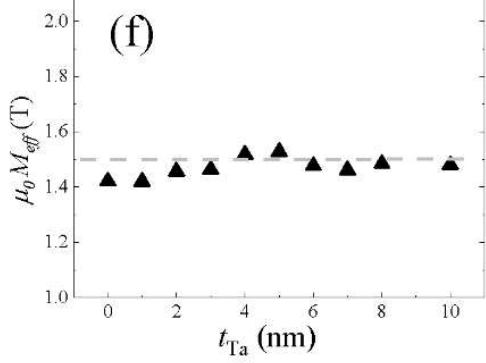

(i)

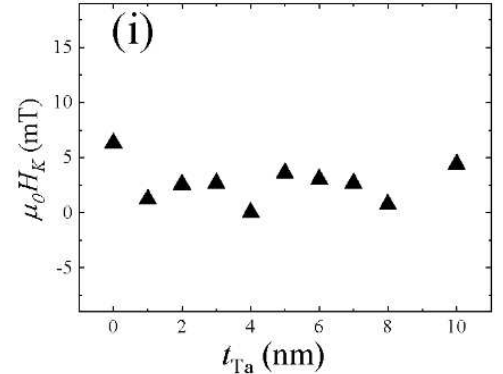

Figure 8: $(\mathrm{a}, \mathrm{b}, \mathrm{c}) \mu_{0} H_{r}$ vs. $f$ plot for the CFB $(4,6,8 \mathrm{~nm}) / \mathrm{Ta}(0-10 \mathrm{~nm})$ bilayers. Symbols are the experimental data and lines are fitted to the experimental data using Eq. (2). (d, e, f) Calculated $\mu_{0} M_{\text {eff }}$ vs. $\mathrm{t}_{\mathrm{Ta}}$ thickness and $(\mathrm{g}, \mathrm{h}, \mathrm{i}) \mu_{0} H_{k}$ vs $\mathrm{t}_{\mathrm{Ta}}$ for the CFB (4, 6, $\left.8 \mathrm{~nm}\right) / \mathrm{Ta}(0-$ $10 \mathrm{~nm}$ ) bilayers. Dashed lines in Fig. 5 (d, e, f) shows the bulk value of effective magnetization of CFB.

The $\mu_{0} \Delta H$ vs. $f$ data for three series are shown in Fig. 9 (a, b, c). Layer stacks are mentioned on top of the corresponding Fig.s. These are fitted with Eq. (4) to obtain effective damping $\alpha_{\text {eff. }}$. The calculated values of $\alpha_{\text {eff }}$ are plotted against $\mathrm{t}_{\mathrm{Ta}}$ in Fig.9 (d, e, f) for all the three series. The variation of $\alpha_{\text {eff }}$ up to $2 \mathrm{~nm}$ thin Ta layer is random (marked by shaded area) and therefore cannot be considered as an effect of spin pumping into Ta. This could possibly be due to the formation of oxide layer of the Ta layer [38]. However, the gradual change is observed in the 
$\alpha_{\text {eff }}$ above $2 \mathrm{~nm}$ thick Ta, which is indicated by the initial enhancement of damping with $\mathrm{t}_{\mathrm{Ta}}$ and then saturation above certain Ta thickness called spin diffusion length in the first two series (i.e., with CFB thickness of 4 and $6 \mathrm{~nm}$ ). This follows the diffusive spin transport and is attributed to the spin pumping effect into the Ta layer from CFB. Further, in CFB $(8 \mathrm{~nm}) / \mathrm{Ta}$ (1-10nm) series depicted in Fig. 5(f), the nature of damping variation is not usual and the damping shows oscillatory behaviour, where $\alpha_{\text {eff }}$ first decreases up to $3 \mathrm{~nm}$ then increases like a normal spin pumping and then above $6 \mathrm{~nm}$ of Ta, it decreases again that could be due to spinback flow. This behaviour is also reported in Py/Ta bilayers [10]. Interesting point to be noted here is that the $\alpha_{\text {eff }}$ shows a FM layer thickness dependence, which has been predicted theoretically [21]. The values of inhomogeneous linewidth contributions of almost all the samples are found to be smaller than $2 \mathrm{mT}$ shown in fig. $9(\mathrm{~g}, \mathrm{~h}, \mathrm{i})$.
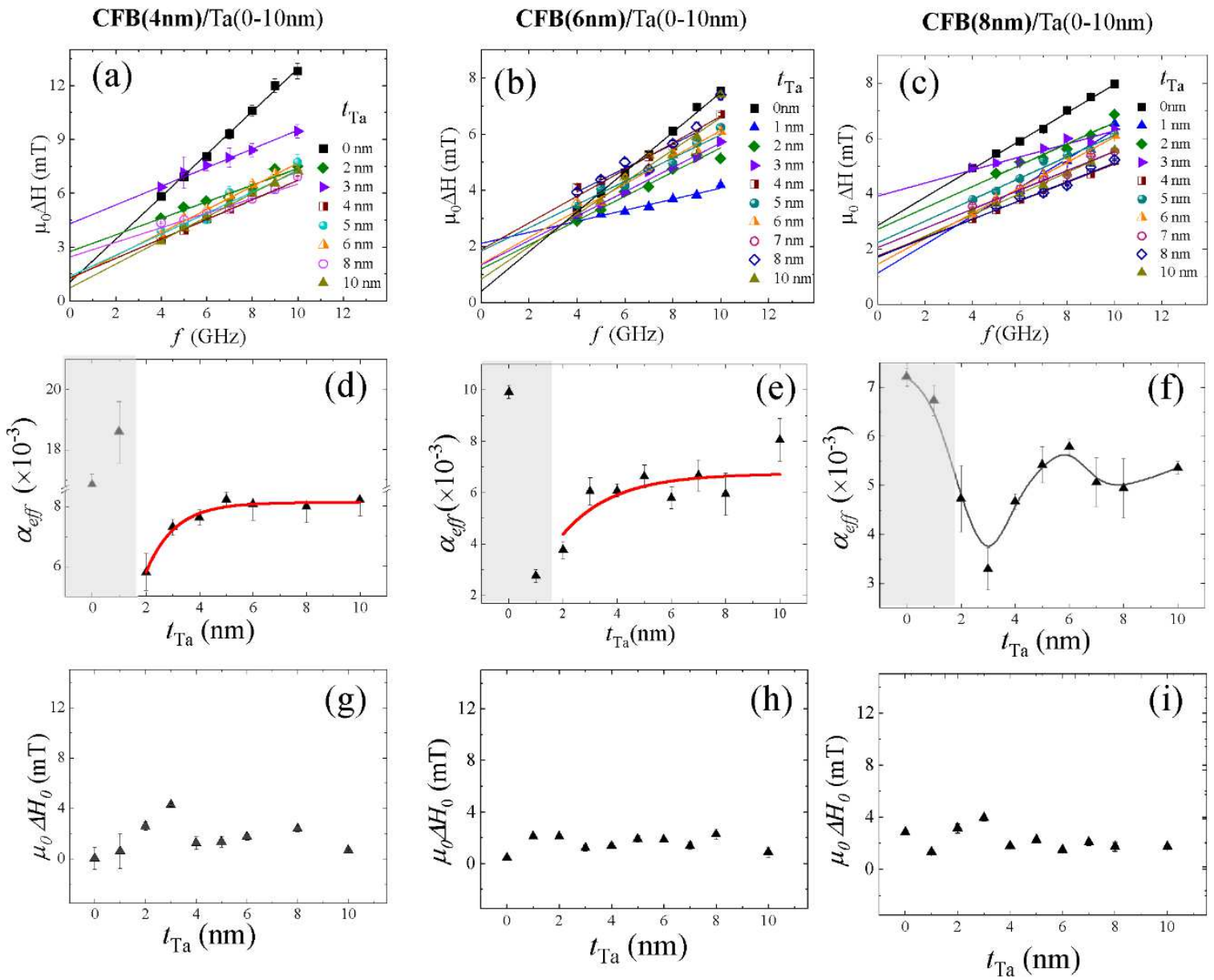

Figure 9: (a-c) Linewidth vs. frequency [lines are fitted to the experimental data using Eq. (4)]. (d-f) Effective damping constant vs $\mathrm{t}_{\mathrm{Ta}}$ [Red line is fitted using Eq. 5.] (g-i) The inhomogeneous linewidth calculated using Eq. 3 by fitting of the data in Fig.9 (a-c). Grey 
shaded region in Fig.9 (d, e and f) indicates the Ta gets affected with oxidation leads to the fluctuation in the damping constant.

In the case of $\mathrm{CFB}(8 \mathrm{~nm}) / \mathrm{Ta}(4 \mathrm{~nm})$ series, the $\alpha_{\text {eff }}$ first gradually decrease on increasing the $\mathrm{t}_{\mathrm{Ta}}$ which has been predicted to be like an anti-damping effect at the CFB/Ta interface. It should not be confused with the anti-damping like torque due to charge current, where the damping is suppressed on applying charge current towards auto-oscillations effect. Here, we have not injected any charge current during the measurements. The observed changes in the effective damping in these bilayers are thus related to the FM layer thickness dependent spin-pumping effects only. The so-called anti-damping effect is generally observed due to non-equilibrium spin accumulation at the FM-HM interface by the combined effects of interfacial Rashba spin orbit splitting and the bulk SOC of HM. It should be noted that Ta possesses low spin flip probability $(<<1)$ and its Fermi-surface is quite different compared with CFB at the interface [39], which contributes to this non equilibrium spin accumulation. However, in the present case, the Rashba spin orbit splitting is probably contributing to the spin accumulation near the interface, compared to that of bulk SOC, since, the anti-damping effect is only active up to $\sim 3$ $\mathrm{nm}$ thick Ta. Earlier report [10] suggests that reduction of damping should sustain up to a thickness double of the spin diffusion length of the HM layer if only bulk SOC is contributing in the spin accumulation. However, if only Rashba SOC contributes to the spin accumulation, it should then be present only up to few nm near the interface. Now, the question arises that why such anti-damping kind of effect is not present in case of the first two series with CFB thickness $4 \mathrm{~nm}$ and $6 \mathrm{~nm}$, respectively. This can be explained in terms of the dependence of Rashba effect on the thickness (conductivity) of FM layer. At the outset of these phenomena, Paul M. Haney et al. theoretically proposed that on increasing the thickness of FM and varying NM layer thickness, the conductivity of the FM plays important role [21] where the spin current generated in the FM/NM bilayer reduces on increasing the NM layer thickness when the large conducting FM is in contact with it due to the increment in anti-damping torque at higher FM thickness. It should be noted that at higher NM thickness the normal spin pumping effect overcome this anti-damping effect. Thus, all these changes in the spin current or damping due to the changes in FM or HM thickness are basically due to the change in the interface (or the electronic structure at the interface) when Rashba spin orbit splitting is predominantly present in thin film.

Further, the quantitative estimation of transfer of spin angular momentum across the $\mathrm{CFB} / \mathrm{Ta}$ bilayer interface i.e., spin pumping is determined by the real part of effective spin- 
mixing conductance. It is defined as the flow of spin angular momentum per unit area through the $\mathrm{CFB} / \mathrm{Ta}$ interface created by the precessing magnetization in the CFB layer. The term effective spin-mixing conductance is being used because it contains both the forward and backflow of spin angular momentum at the CFB/Ta bilayer interface. To determine the real part of the spin-mixing conductance, the obtained $\mathrm{t}_{\mathrm{Ta}}$ dependent Gilbert damping is fitted with the ballistic spin transport model [40],

$$
\alpha_{e f f}-\alpha_{C F B}=\operatorname{Re}\left(g_{e f f}^{\uparrow \downarrow}\right) \frac{g \mu_{B}}{4 \pi M_{S}} \frac{1}{t_{C F B}}\left(1-e^{-\frac{2 t_{T a}}{\lambda_{d}}}\right)
$$

Where $\operatorname{Re}\left(g_{\text {eff }}^{\uparrow \downarrow}\right)$ is the effective spin mixing conductance given in units of $\mathrm{m}^{-2}$. Fig. 9 (d, e, f) show the variation observed in $\alpha_{e f f}$ with $\mathrm{t}_{\mathrm{Ta}}$ for all the bilayer series of different $\mathrm{t}_{\mathrm{CFB}}$ and also the fitted using to Eq. 5. The spin diffusion lengths have been estimated to be $2.96( \pm 0.25) \mathrm{nm}$, $3.81( \pm 0.74) \mathrm{nm}$ for series CFB $\left(\mathrm{t}_{\mathrm{CFB}}=4\right) / \mathrm{Ta}\left(\mathrm{t}_{\mathrm{Ta}}=0-10 \mathrm{~nm}\right)$, and CFB $\left(\mathrm{t}_{\mathrm{CFB}}=6\right) / \mathrm{Ta}\left(\mathrm{t}_{\mathrm{Ta}}=0-\right.$ $10 \mathrm{~nm})$, respectively. These spin diffusion lengths values also include the $\sim 2 \mathrm{~nm}$ oxide layer of Ta as shown in XRR measurement. Further, the spin mixing conductance values have been obtained for the two series are $26.5( \pm 0.4) \mathrm{nm}^{-2}$ and $21.4( \pm 0.3) \mathrm{nm}^{-2}$, respectively for CFB $(4 \mathrm{~nm}) / \mathrm{Ta}$, and CFB $(6 \mathrm{~nm}) / \mathrm{Ta}$ bilayer series. However, in the $\mathrm{CFB}(8 \mathrm{~nm}) / \mathrm{Ta}$ series, the mixing conductance is not possible to obtain by fitting due to its oscillatory behaviour. Important to note that we do not intend to quantitatively estimate the spin diffusion length and spin mixing conductance, but how the nature of spin transport through the interface changes as a function of FM thickness, is the prime motive of our present study. It is quite visible that the behaviour of damping modulation is correlated with the thickness of the FM layer in the bilayers.

\section{Conclusions}

We have reported the ferromagnetic thickness dependent spin pumping in three different CFB $(4,6 \& 8 \mathrm{~nm}) / \mathrm{Ta}(1-10 \mathrm{~nm}$ in steps of $1 \mathrm{~nm})$ bilayer series. It has been observed that in the first two series with fixed CFB thickness of 4 and $6 \mathrm{~nm}$, normal spin pumping behaviour occurred but anomalous behaviour observed for the bilayer series with $8 \mathrm{~nm}$ thick CFB. The origin behind such anomalous behaviour is possibly related to the large conductivity of CFB together with large Rashba SOC. This particular series also exhibited the spin back-flow at the CFB/Ta interface. From this analysis, it is understood that the thickness of ferromagnetic layer plays an important role for spin current generation. The study suggests that the FM layer thickness is crucial to study the spin current behaviour in spintronic applications. 


\section{Acknowledgements}

S. Hait acknowledges the Ministry of Education, Government of India, for the financial support. S. Husian acknowledges the Department of Science and Technology, Government of India for providing the INSPIRE Fellowship (IF140093). Carl Tryggers Stiftelse for Vetenskaplig Forskning (grant no: CTS 17:450) is gratefully acknowledged for the support.

\section{References}

[1] M. Jamali, A. Klemm, J.P. Wang, Precessional magnetization induced spin current from CoFeB into Ta, Appl. Phys. Lett. 103 (2013). https://doi.org/10.1063/1.4853195.

[2] Y. Tserkovnyak, A. Brataas, G.E.W. Bauer, Spin pumping and magnetization dynamics in metallic multilayers, Phys. Rev. B. 66 (2002) 224403. https://doi.org/10.1103/PhysRevB.66.224403.

[3] A. Conca, B. Heinz, M.R. Schweizer, S. Keller, E.T. Papaioannou, B. Hillebrands, Lack of correlation between the spin-mixing conductance and the inverse spin Hall effect generated voltages in CoFeB/Pt and CoFeB/Ta bilayers, Phys. Rev. B. 95 (2017) 174426. https://doi.org/10.1103/PhysRevB.95.174426.

[4] Z. Zhu, B. Zhao, W. Zhu, M. Tang, Y. Ren, Q.Y. Jin, Z. Zhang, Annealing effect and interlayer modulation on magnetic damping of $\mathrm{CoFeB} /$ interlayer/Pt thin films, Appl. Phys. Lett. 113 (2018). https://doi.org/10.1063/1.5050445.

[5] D.-J. Kim, S.-I. Kim, S.-Y. Park, K.-D. Lee, B.-G. Park, Ferromagnetic resonance spin pumping in $\mathrm{CoFeB}$ with highly resistive non-magnetic electrodes, Curr. Appl. Phys. 14 (2014) 1344-1348. https://doi.org/10.1016/j.cap.2014.07.013.

[6] A. Kumar, S. Akansel, H. Stopfel, M. Fazlali, J. Akerman, R. Brucas, P. Svedlindh, Spin transfer torque ferromagnetic resonance induced spin pumping in the $\mathrm{Fe} / \mathrm{Pd}$ bilayer system, Phys. Rev. B. 95 (2017) 6-12. https://doi.org/10.1103/PhysRevB.95.064406.

[7] C. He, A. Navabi, Q. Shao, G. Yu, D. Wu, W. Zhu, C. Zheng, X. Li, Q.L. He, S.A. Razavi, K.L. Wong, Z. Zhang, P.K. Amiri, K.L. Wang, Spin-torque ferromagnetic resonance measurements utilizing spin Hall magnetoresistance in $\mathrm{W} / \mathrm{Co} 40 \mathrm{Fe}_{40} \mathrm{~B}_{20} / \mathrm{MgO}$ structures, Appl. Phys. Lett. 109 (2016) 202404. https://doi.org/10.1063/1.4967843. 
[8] D. Jhajhria, N. Behera, D.K. Pandya, S. Chaudhary, Dependence of spin pumping in W/CoFeB heterostructures on the structural phase of tungsten, Phys. Rev. B. 99 (2019) 1-8. https://doi.org/10.1103/PhysRevB.99.014430.

[9] S.N. Panda, S. Majumder, A. Bhattacharyya, S. Dutta, S. Choudhury, A. Barman, Structural Phase Dependent Giant Interfacial Spin Transparency in W/CoFeB Thin Film Heterostructure, (2020). http://arxiv.org/abs/2009.14143.

[10] N. Behera, S. Chaudhary, D.K. Pandya, Anomalous anti-damping in sputtered $\beta-T a / P y$ bilayer system, Sci. Rep. 6 (2016) 19488. https://doi.org/10.1038/srep19488.

[11] S. Akansel, A. Kumar, N. Behera, S. Husain, R. Brucas, S. Chaudhary, P. Svedlindh, Thickness-dependent enhancement of damping in $\mathrm{Co}_{2} \mathrm{FeAl} / \beta$-Ta thin films, Phys. Rev. B. 97 (2018) 1-8. https://doi.org/10.1103/PhysRevB.97.134421.

[12] A. Kumar, R. Gupta, S. Husain, N. Behera, S. Hait, S. Chaudhary, R. Brucas, P. Svedlindh, Spin pumping and spin torques in interfacially tailored $\mathrm{Co}_{2} \mathrm{FeAl} / \beta-\mathrm{Ta}$ layers, Phys. Rev. B. 100 (2019) 1-8. https://doi.org/10.1103/PhysRevB.100.214433.

[13] O. Mosendz, V. Vlaminck, J.E. Pearson, F.Y. Fradin, G.E.W. Bauer, S.D. Bader, A. Hoffmann, Detection and quantification of inverse spin Hall effect from spin pumping in permalloy/normal metal bilayers, Phys. Rev. B. 82 (2010) 214403. https://doi.org/10.1103/PhysRevB.82.214403.

[14] S. Husain, A. Kumar, V. Barwal, N. Behera, S. Akansel, P. Svedlindh, S. Chaudhary, PHYSICAL REVIEW B 97 , 064420 ( 2018 ) Spin pumping in ion-beam sputtered Co 2 FeAl / Mo bilayers : Interfacial Gilbert damping, Phys. Rev. B. 064420 (2018) 2-9. https://doi.org/10.1103/PhysRevB.97.064420.

[15] M. Morota, Y. Niimi, K. Ohnishi, D.H. Wei, T. Tanaka, H. Kontani, T. Kimura, Y. Otani, Indication of intrinsic spin Hall effect in $4 d$ and 5d transition metals, Phys. Rev. B. 83 (2011) 174405. https://doi.org/10.1103/PhysRevB.83.174405.

[16] S.D. Stranks, P. Plochocka, The influence of the Rashba effect, Nat. Mater. 17 (2018) 381-382. https://doi.org/10.1038/s41563-018-0067-8.

[17] K.-W. Kim, S.-M. Seo, J. Ryu, K.-J. Lee, H.-W. Lee, Magnetization dynamics induced by in-plane currents in ultrathin magnetic nanostructures with Rashba spin-orbit coupling, Phys. Rev. B. 85 (2012) 180404. https://doi.org/10.1103/PhysRevB.85.180404. 
[18] N. Hwayong, L. S., H.C. S., C.K. Hyoung, N.P. L., W.W. K., Measurement of the Intrinsic Anomalous Hall Effect in a 2D Hole System with Rashba Spin-orbit Coupling, J. Korean Phys. Soc. 57 (2010) 1933. https://doi.org/10.3938/jkps.57.1933.

[19] G. Allen, S. Manipatruni, D.E. Nikonov, M. Doczy, I.A. Young, Experimental demonstration of the coexistence of spin Hall and Rashba effects in b-Ta/ferromagnets bilayers, Phys. Rev. B. 91 (2015) 144412. https://doi.org/10.1103/PhysRevB.91.144412.

[20] Q. Shao, G. Yu, Y.-W. Lan, Y. Shi, M.-Y. Li, C. Zheng, X. Zhu, L.-J. Li, P.K. Amiri, K.L. Wang, Strong Rashba-Edelstein Effect-Induced Spin-Orbit Torques in Monolayer Transition Metal Dichalcogenide/Ferromagnet Bilayers, Nano Lett. 16 (2016) 7514-7520. https://doi.org/10.1021/acs.nanolett.6b03300.

[21] P.M. Haney, H.-W. Lee, K.-J. Lee, A. Manchon, M.D. Stiles, Current induced torques and interfacial spin-orbit coupling: Semiclassical modeling, Phys. Rev. B. 87 (2013) 174411. https://doi.org/10.1103/PhysRevB.87.174411.

[22] S.X. Huang, T.Y. Chen, C.L. Chien, Spin polarization of amorphous CoFeB determined by point-contact Andreev reflection, Appl. Phys. Lett. 92 (2008). https://doi.org/10.1063/1.2949740.

[23] F. Te Yuan, Y.H. Lin, J.K. Mei, J.H. Hsu, P.C. Kuo, Effect of thickness of MgO, Co$\mathrm{Fe}-\mathrm{B}$, and Ta layers on perpendicular magnetic anisotropy of $\left[\mathrm{Ta} / \mathrm{Co}_{60} \mathrm{Fe}_{20} \mathrm{~B}{ }_{20} / \mathrm{MgO}\right]_{5}$ multilayered films, J. Appl. Phys. 111 (2012) 7-10. https://doi.org/10.1063/1.3673408.

[24] J.M. Shaw, H.T. Nembach, M. Weiler, T.J. Silva, M. Schoen, J.Z. Sun, D.C. Worledge, Perpendicular Magnetic Anisotropy and Easy Cone State in $\mathrm{Ta} / \mathrm{Co}_{60} \mathrm{Fe}_{20} \mathrm{~B}_{20} / \mathrm{MgO}$, IEEE Magn. Lett. 6 (2015). https://doi.org/10.1109/LMAG.2015.2438773.

[25] S. Hait, S. Husain, V. Barwal, N.K. Gupta, L. Pandey, P. Svedlindh, S. Chaudhary, Comparison of high temperature growth versus post-deposition in situ annealing in attaining very low Gilbert damping in sputtered $\mathrm{Co}_{2} \mathrm{Fe} A l$ Heusler alloy films, J. Magn. Magn. Mater. 519 (2021) 167509. https://doi.org/10.1016/j.jmmm.2020.167509.

[26] S. Husain, N.K. Gupta, V. Barwal, S. Chaudhary, Magneto-optical properties of CoFeB ultrathin films: Effect of Ta buffer and capping layer, in: AIP Conf. Proc., 2018: p. 120048. https://doi.org/10.1063/1.5033113. 
[27] Y.T. Chen, C.C. Chang, Effect of grain size on magnetic and nanomechanical properties of $\mathrm{Co}_{60} \mathrm{Fe}_{20} \mathrm{~B}_{20}$ thin films, J. Alloys Compd. 498 (2010) 113-117. https://doi.org/10.1016/j.jallcom.2010.03.141.

[28] L. Kipgen, H. Fulara, M. Raju, S. Chaudhary, In-plane magnetic anisotropy and coercive field dependence upon thickness of CoFeB, J. Magn. Magn. Mater. 324 (2012) 3118-3121. https://doi.org/10.1016/j.jmmm.2012.05.012.

[29] A. Jiang, T.A. Tyson, L. Axe, L. Gladczuk, M. Sosnowski, P. Cote, The structure and stability of $\beta$-Ta thin films, Thin Solid Films. 479 (2005) 166-173. https://doi.org/10.1016/j.tsf.2004.12.006.

[30] E.A.I. Ellis, M. Chmielus, S.P. Baker, Effect of sputter pressure on Ta thin films: Beta phase formation, texture, and stresses, Acta Mater. 150 (2018) 317-326. https://doi.org/10.1016/j.actamat.2018.02.050.

[31] M. Cecot, Ł. Karwacki, W. Skowroński, J. Kanak, J. Wrona, A. Zywczak, L. Yao, S. Dijken, J. Barnaś, T. Stobiecki, Influence of intermixing at the $\mathrm{Ta} / \mathrm{CoFeB}$ interface on spin Hall angle in Ta/CoFeB/MgO heterostructures, Sci. Rep. 7 (2017) 1-11. https://doi.org/10.1038/s41598-017-00994-z.

[32] M. Raju, N. Behera, D.K. Pandya, S. Chaudhary, Magnetization dynamics and interface studies in ion-beam sputtered $\mathrm{Si} / \mathrm{CoFeB}(8) / \mathrm{MgO}(4) / \mathrm{CoFeB}(8) / \mathrm{Ta}(5)$ structures, J. Appl. Phys. 115 (2014) 17D127. https://doi.org/10.1063/1.4863804.

[33] L. Jin, K. Jia, D. Zhang, B. Liu, H. Meng, X. Tang, Z. Zhong, H. Zhang, Effect of Interfacial Roughness Spin Scattering on the Spin Current Transport in YIG/NiO/Pt Heterostructures, ACS Appl. Mater. Interfaces. 11 (2019) 35458-35467. https://doi.org/10.1021/acsami.9b12125.

[34] L.H. Bai, Y.S. Gui, A. Wirthmann, E. Recksiedler, N. Mecking, C.-M. Hu, Z.H. Chen, S.C. Shen, The rf magnetic-field vector detector based on the spin rectification effect, Appl. Phys. Lett. 92 (2008) 032504. https://doi.org/10.1063/1.2837180.

[35] C. Kittel, On the Theory of Ferromagnetic Resonance Absorption, Phys. Rev. 73 (1948) 155-161. https://doi.org/10.1103/PhysRev.73.155.

[36] G. Lu, X. Huang, S. Fan, W. Ling, M. Liu, J. Li, L. Jin, L. Pan, Temperature-and thickness -dependent dynamic magnetic properties of sputtered $\mathrm{CoFeB} / \mathrm{Ta}$ bilayer films, J. Alloys Compd. 753 (2018) 475-482. 
https://doi.org/10.1016/j.jallcom.2018.04.262.

[37] A. Gayen, R. Modak, A. Srinivasan, V.V. Srinivasu, P. Alagarsamy, Thickness dependent magneto-static and magneto-dynamic properties of $\mathrm{CoFeB}$ thin films, J. Vac. Sci. Technol. A. 37 (2019) 031513. https://doi.org/10.1116/1.5091675.

[38] N. Behera, P. Guha, D.K. Pandya, S. Chaudhary, Capping Layer (CL) Induced Antidamping in CL/Py/ $\beta-W$ System (CL: Al, $\beta$-Ta, $\mathrm{Cu}, \beta-\mathrm{W}$ ), ACS Appl. Mater. Interfaces. 9 (2017) 31005-31017. https://doi.org/10.1021/acsami.7b06991.

[39] S.N. Panda, S. Mondal, J. Sinha, S. Choudhury, A. Barman, All-optical detection of interfacial spin transparency from spin pumping in $\beta-\mathrm{Ta} / \mathrm{CoFeB}$ thin films, Sci. Adv. 5 (2019) eaav7200. https://doi.org/10.1126/sciadv.aav7200.

[40] J.M. Shaw, H.T. Nembach, T.J. Silva, Determination of spin pumping as a source of linewidth in sputtered $\mathrm{CoFe} / \mathrm{Pd}$ multilayers by use of broadband ferromagnetic resonance spectroscopy, Phys. Rev. B. 85 (2012) 054412. https://doi.org/10.1103/PhysRevB.85.054412. 
Figures

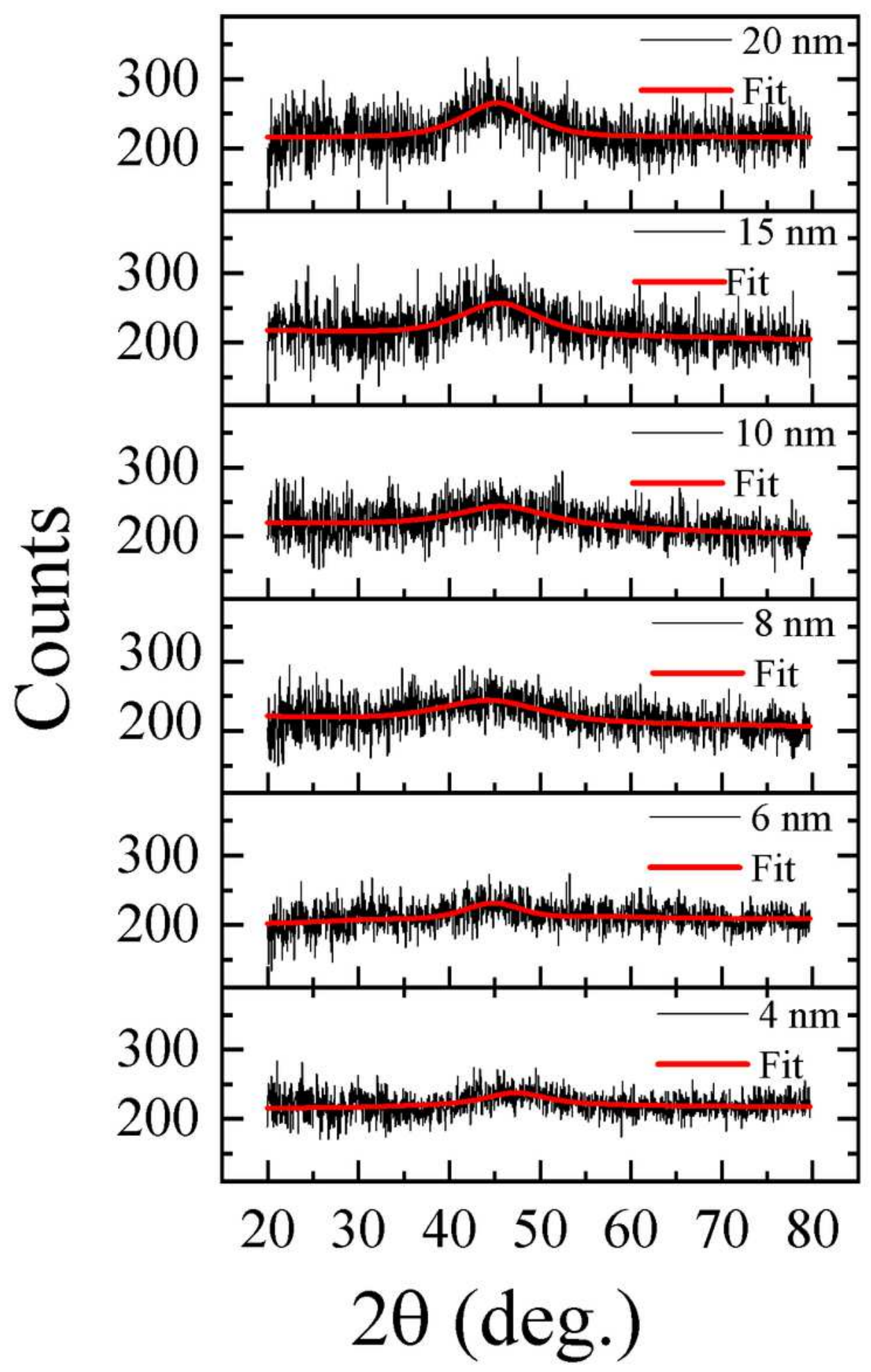

Figure 1

GIXRD spectra of CFB films of different indicated thicknesses tCFB in nm. 


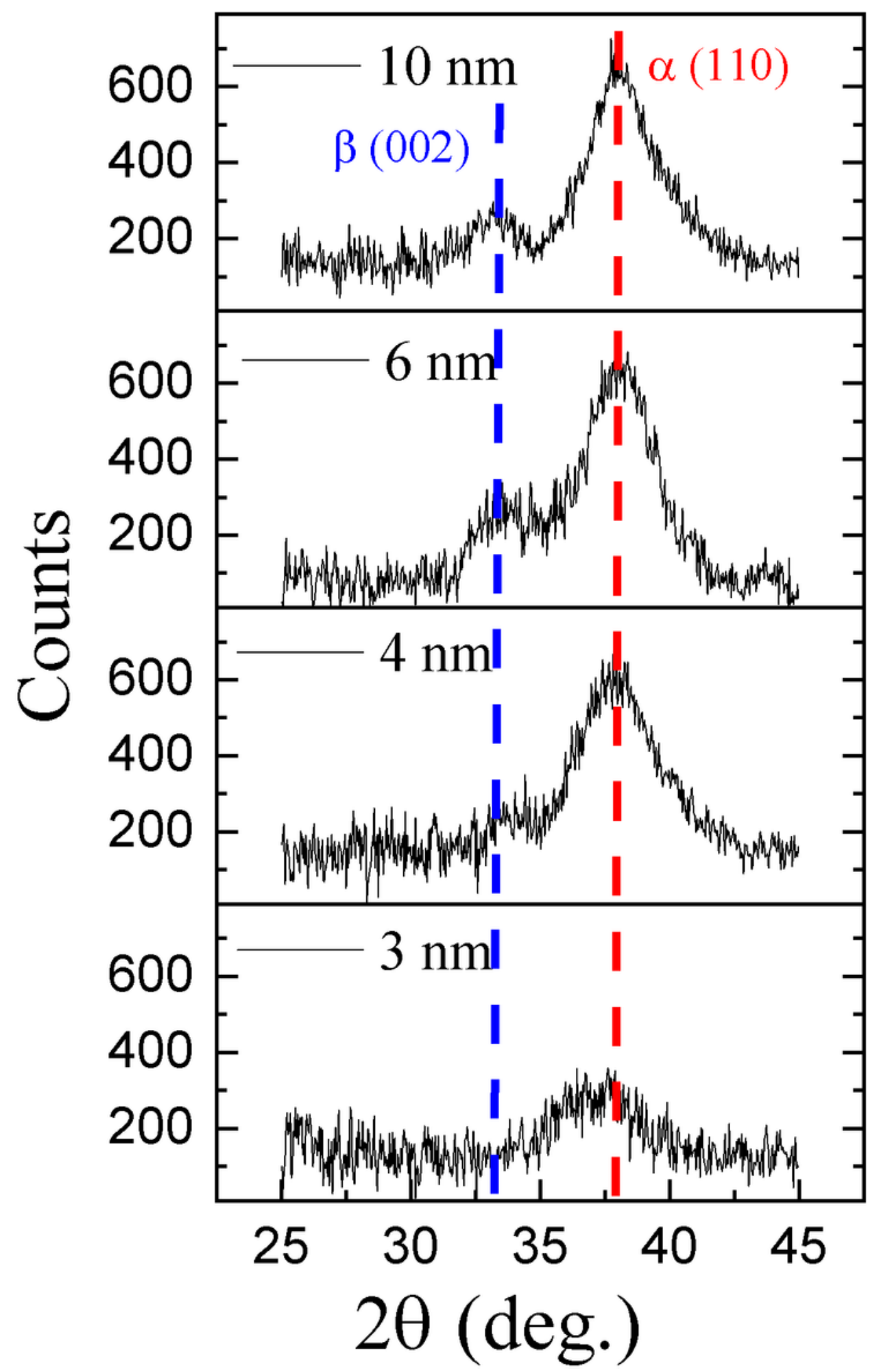

Figure 2

GIXRD spectra of CFB ( $4 \mathrm{~nm}) / \mathrm{Ta}(\mathrm{tTa}=10,6,4,3 \mathrm{~nm})$ bilayers. The bleu (red) dash lines mark the $2 \theta$ positions of the most intense peaks of the $\beta$-Ta $(a-T a)$ phase as per JCPDS standards. 


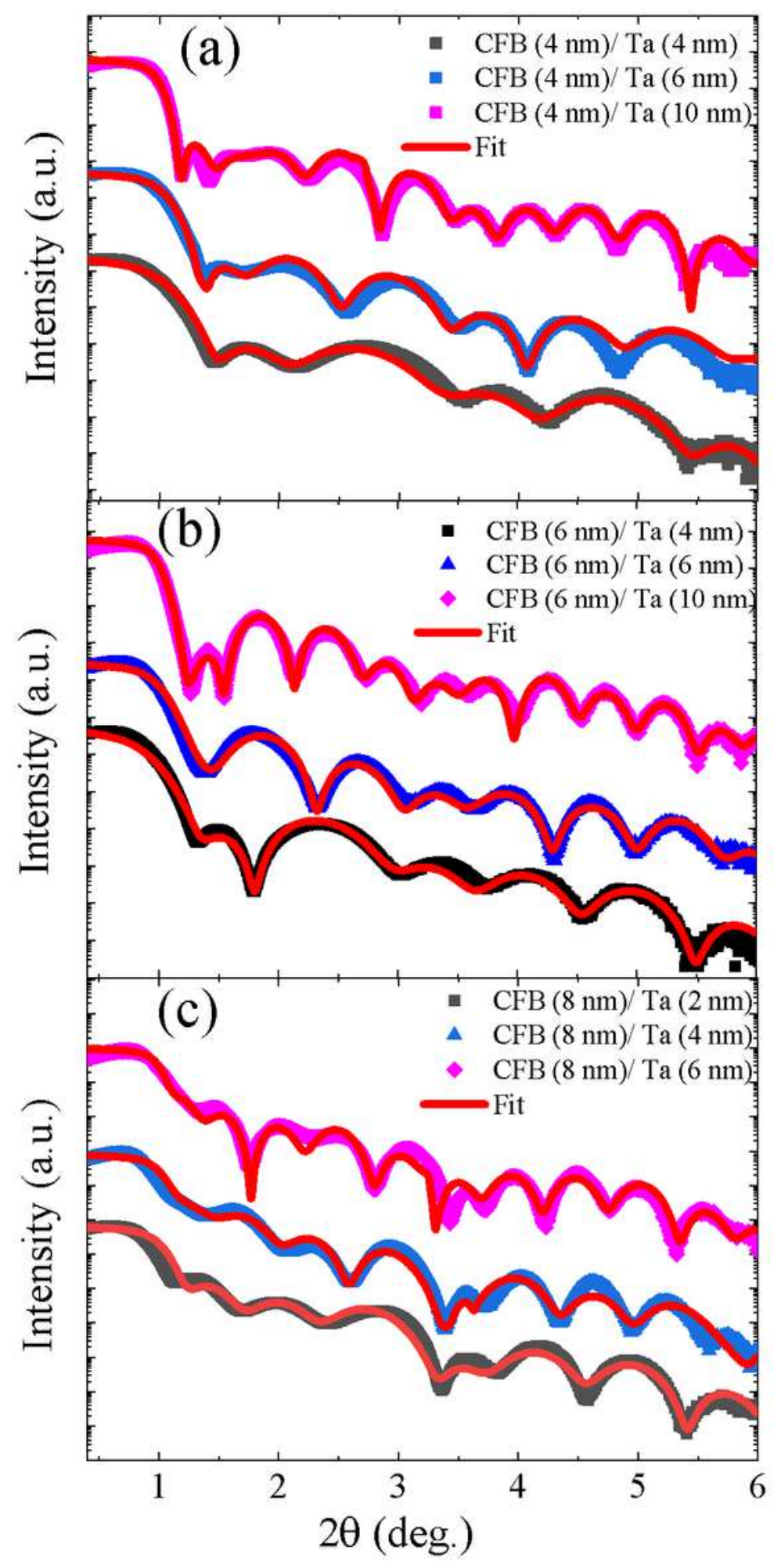

Figure 3

XRR spectra of (a) CFB (4 nm)/ Ta $(4,6,10 \mathrm{~nm})$, (b) CFB $(6 \mathrm{~nm}) / \mathrm{Ta}(4,6,10 \mathrm{~nm})$ and (c) CFB $(8 \mathrm{~nm}) / \mathrm{Ta}$ $(4,6,8 \mathrm{~nm})$. Red lines represent the fitted spectra. 

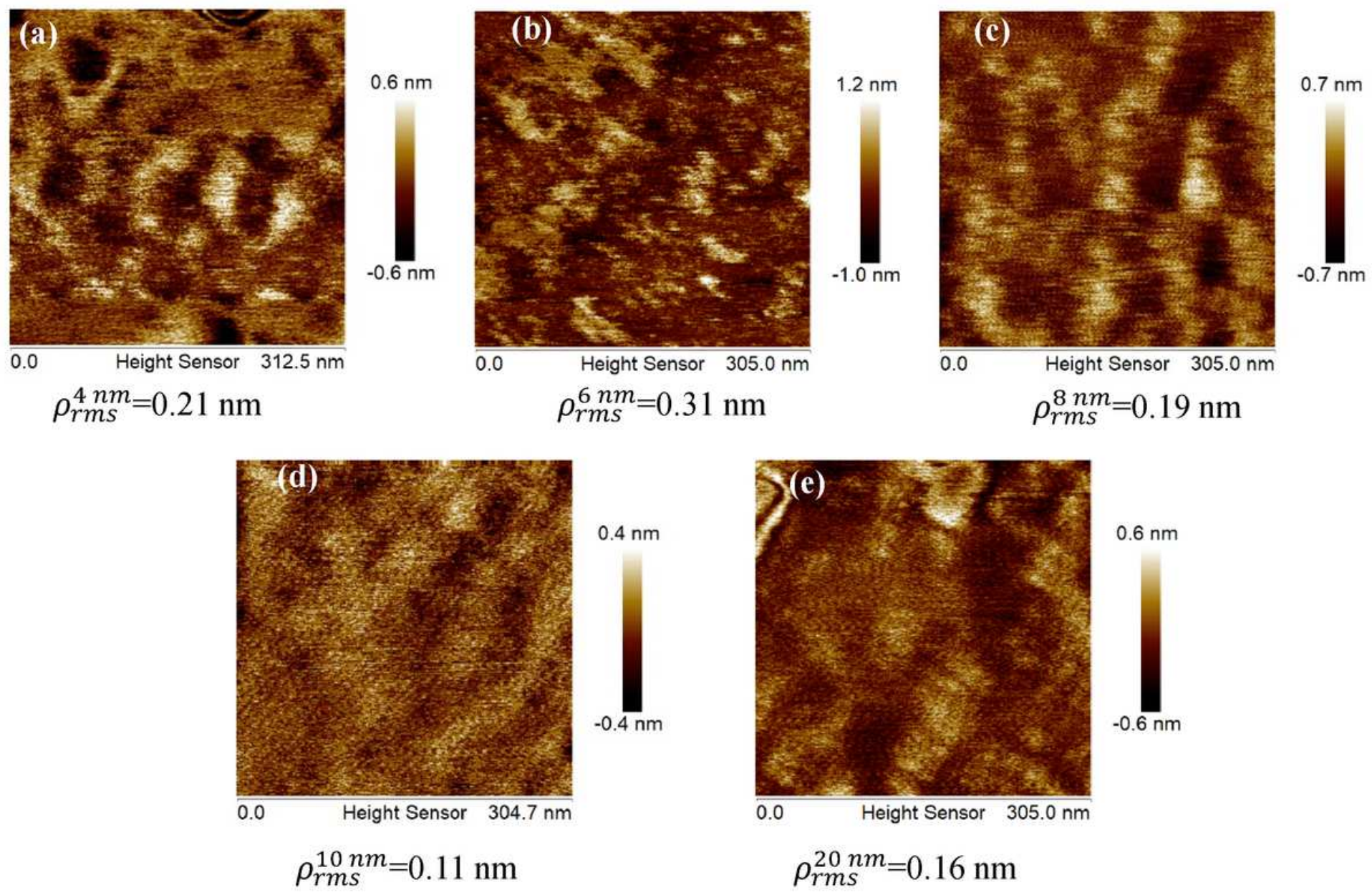

\section{Figure 4}

Topography of single layer CFB thin film of different thickness, (a) $4 \mathrm{~nm}$, (b) $6 \mathrm{~nm}$, (c) $8 \mathrm{~nm}$, (d) $10 \mathrm{~nm}$, and (e) $20 \mathrm{~nm}$. 

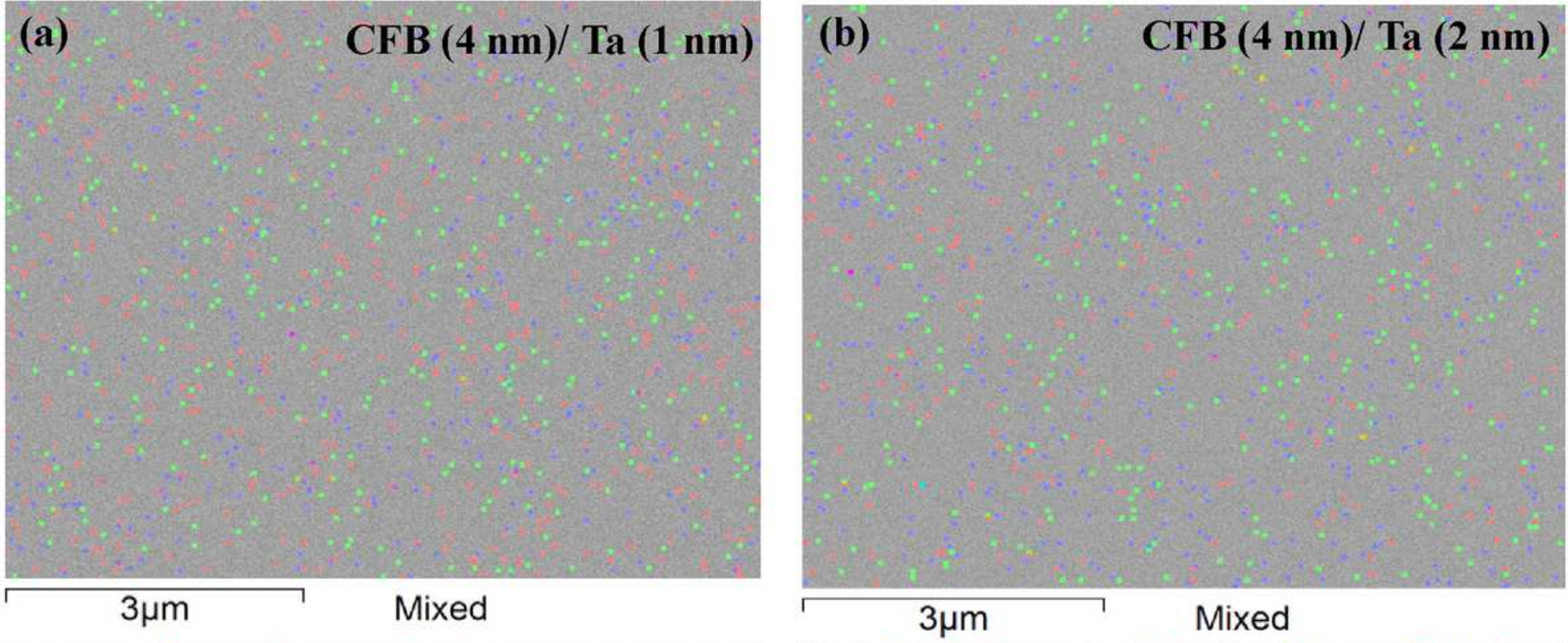

\section{Figure 5}

EDX mapping of (a) CFB (4 nm)/Ta (1 nm), (b) CFB (4 nm)/Ta (2 nm), (c) CFB (4 nm)/Ta (3 nm) and (d) CFB (4 nm)/Ta (4 nm), respectively. The red, blue and green points represent $\mathrm{Ta}$, Co and Fe respectively. These images clearly reveal the composition homogeneity in these samples. 

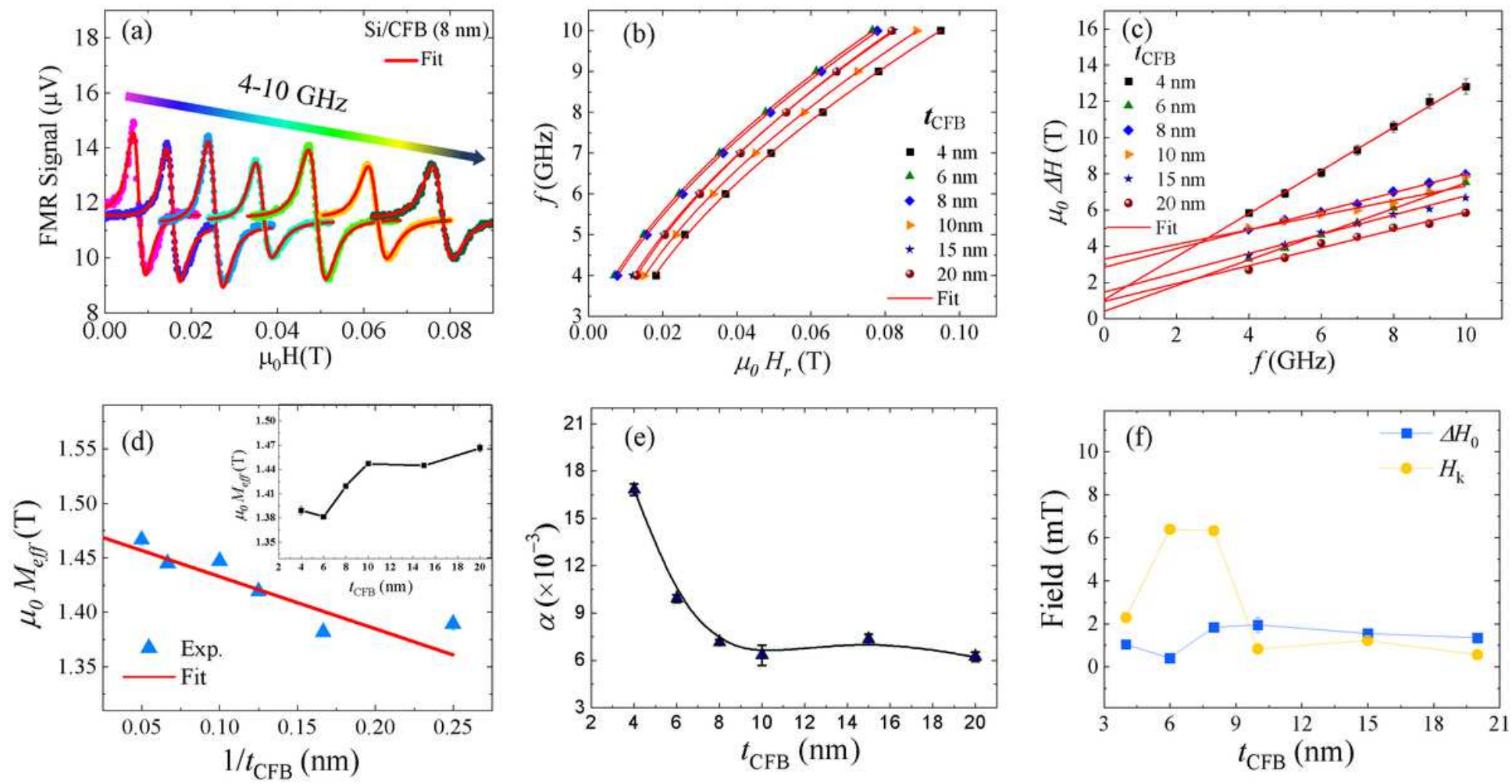

Figure 6

(a) FMR spectra of $8 \mathrm{~nm}$ single layer CFB thin film. Filled circles are the experimental data points and red lines shows the fitting using Eq. (1). (b and c) Variation in resonance field and linewidth, respectively, as a function of microwave frequency. ( $d$, e and $f$ ) Calculated effective magnetization, anisotropy field and inhomogeneous linewidth of the films as a function of thickness of CFB layer.
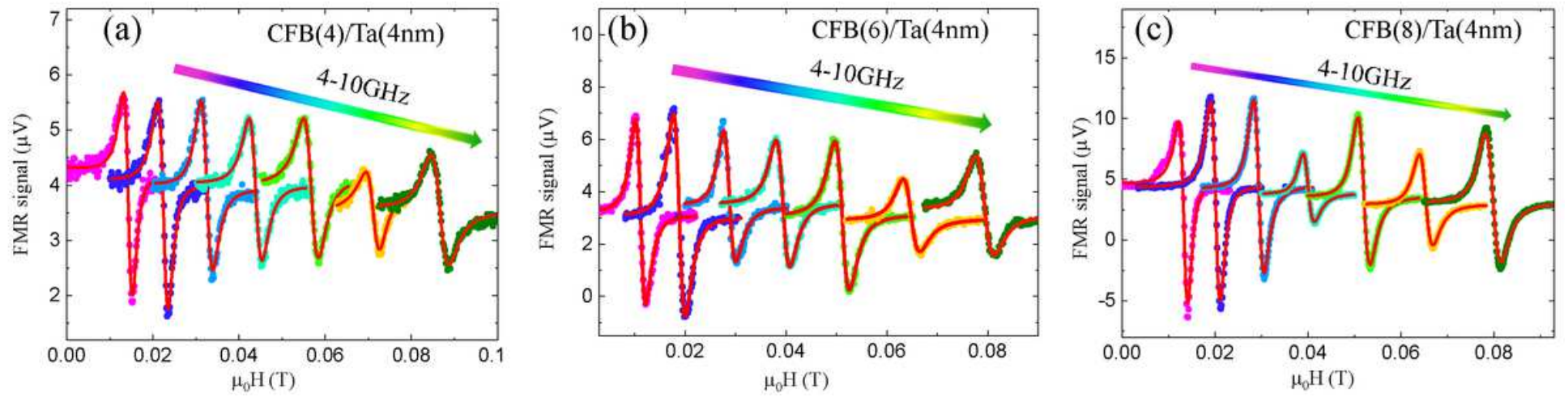

Figure 7

FMR spectra recorded on (a) CFB(4nm)/Ta(4nm), (b) CFB(6nm)/ Ta (4nm) and (c) CFB(8nm)/ Ta (4nm) bilayer thin films. Filled circles are the experimental data points and red lines shows the fitting using Eq. (1). 
CFB(4nm)/Ta(0-10nm)
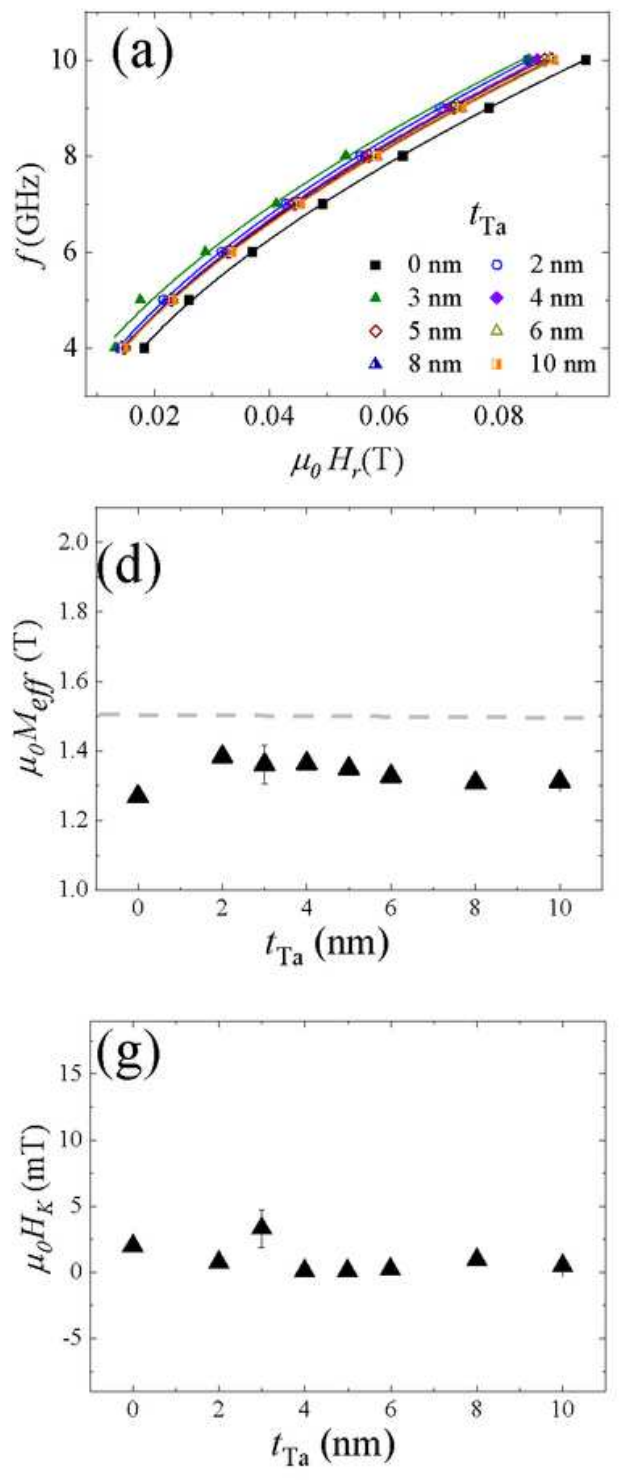

CFB(6nm)/Ta(0-10nm)
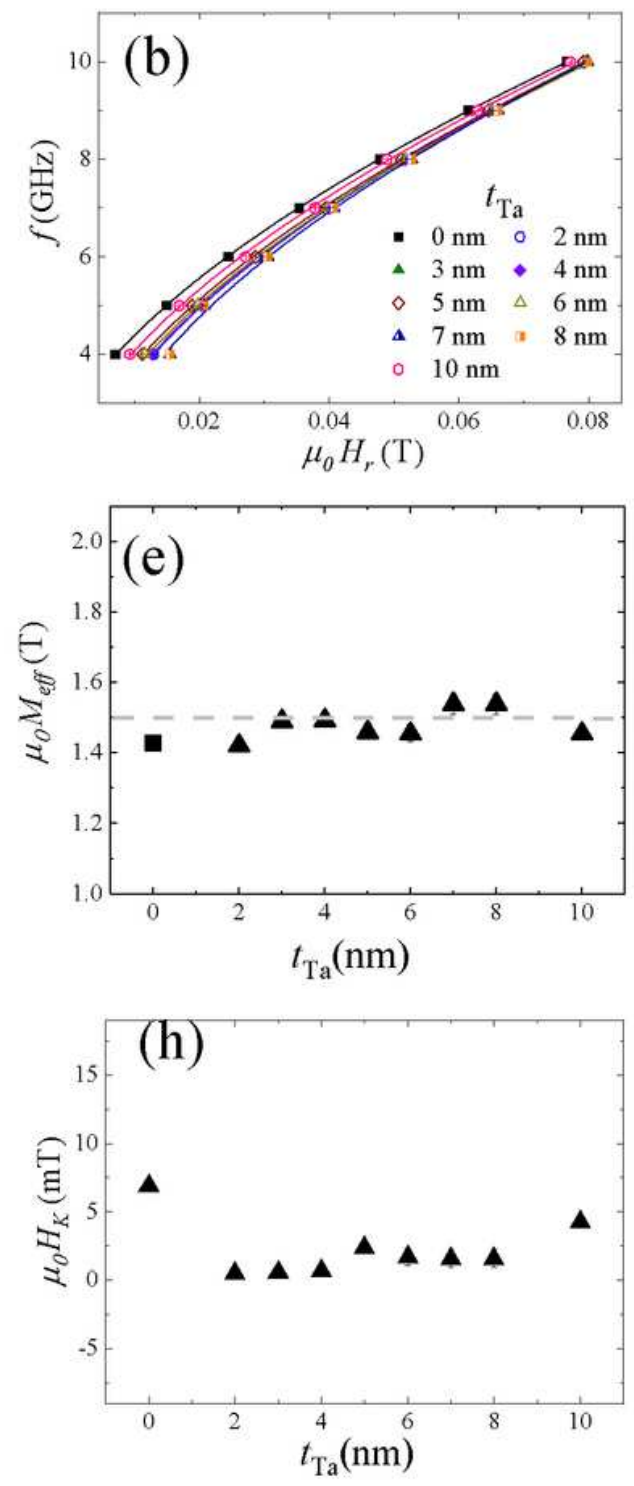

CFB(8nm)/Ta(0-10nm)
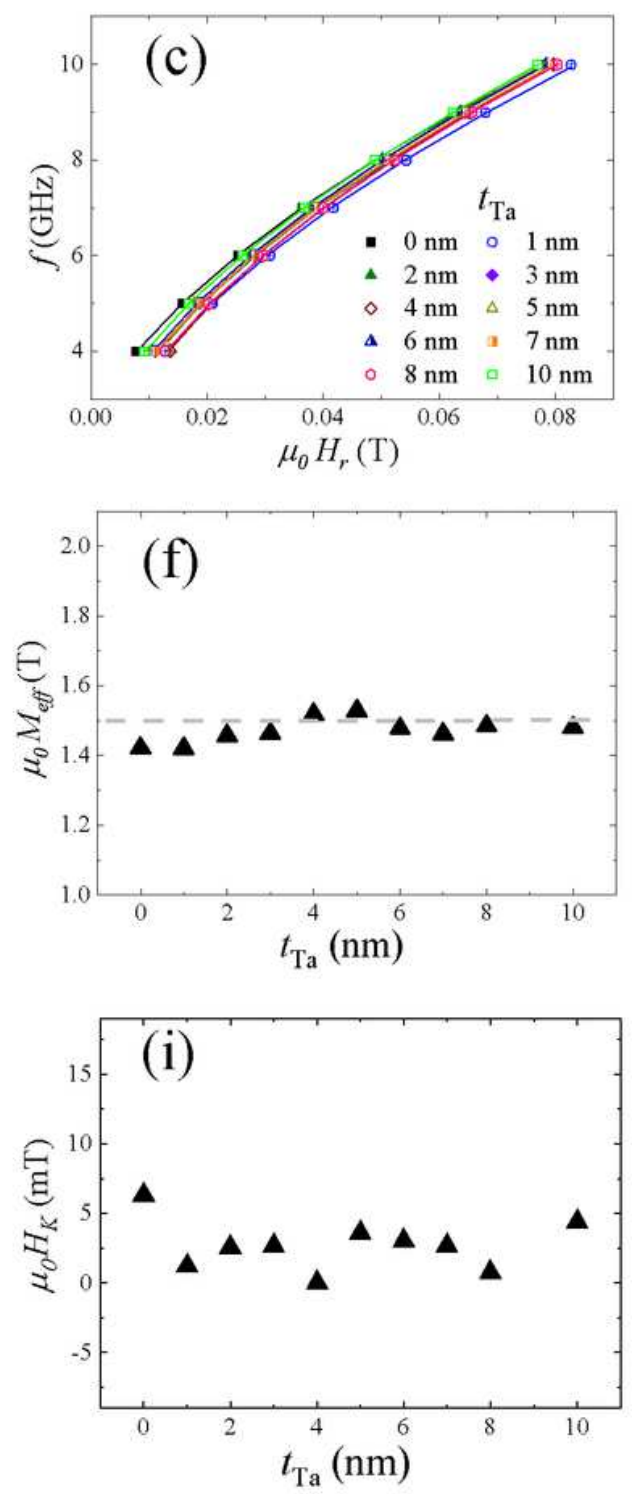

Figure 8

$(a, b, c) \mu 0 \mathrm{Hr}$ vs. $f$ plot for the CFB $(4,6,8 \mathrm{~nm}) / \mathrm{Ta}(0-10 \mathrm{~nm})$ bilayers. Symbols are the experimental data and lines are fitted to the experimental data using Eq. (2). (d, e, f) Calculated $\mu 0$ Meff vs tTa thickness and $(\mathrm{g}, \mathrm{h}, \mathrm{i}) \mu \mathrm{OHk}$ vs tTa for the CFB $(4,6,8 \mathrm{~nm}) / \mathrm{Ta}(0-10 \mathrm{~nm})$ bilayers. Dashed lines in Fig. $5(\mathrm{~d}, \mathrm{e}, \mathrm{f})$ shows the bulk value of effective magnetization of CFB. 
CFB(4nm)/Ta(0-10nm)
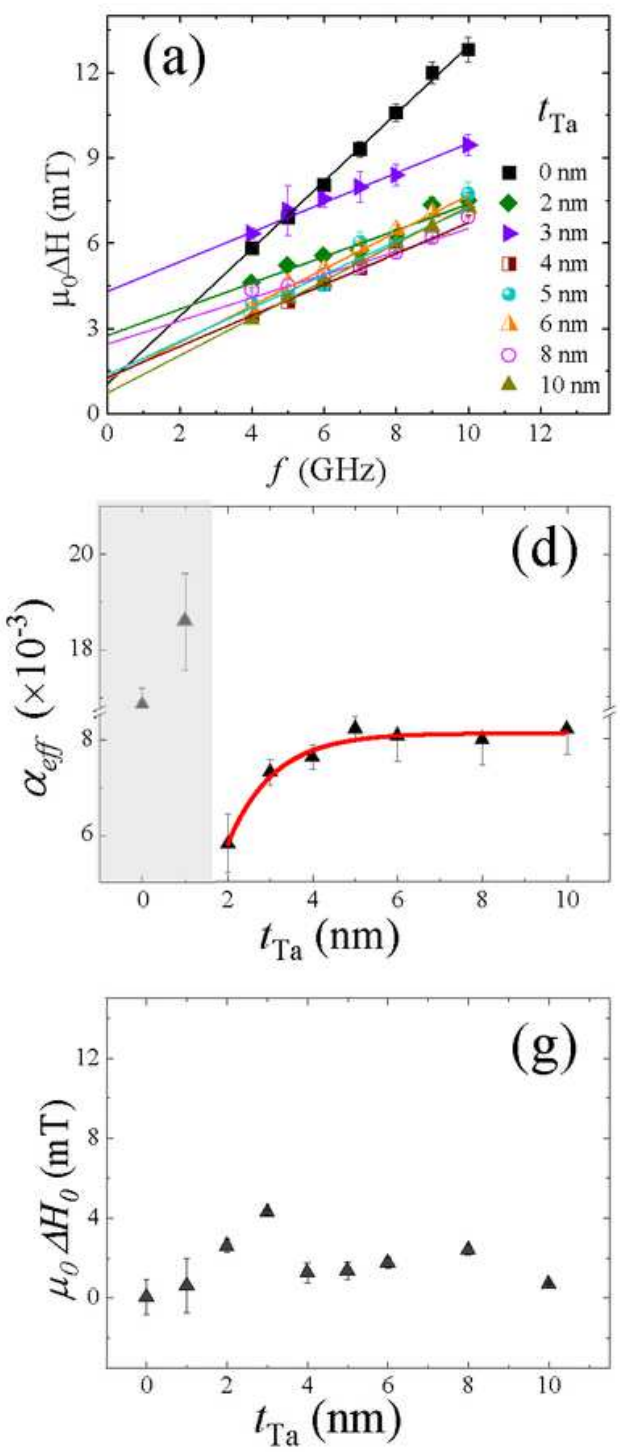

CFB(6nm)/Ta(0-10nm)
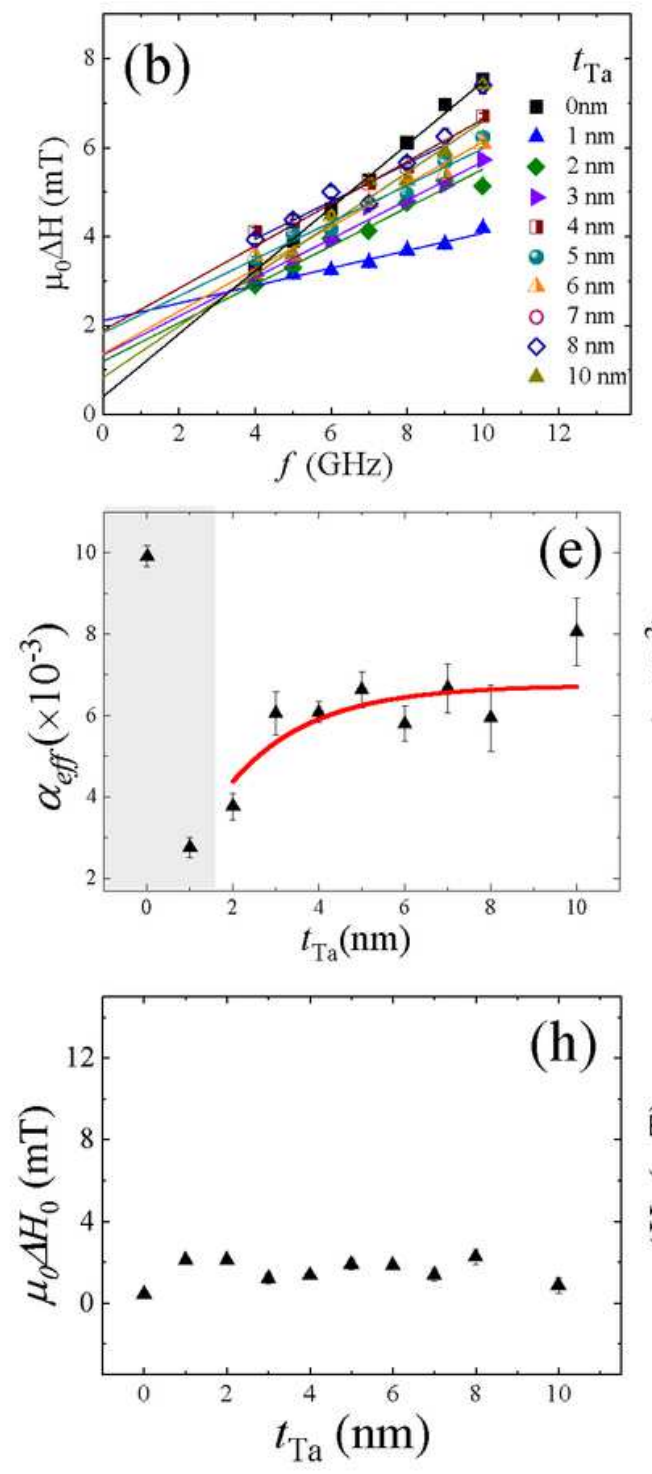

$\operatorname{CFB}(8 \mathrm{~nm}) / \mathrm{Ta}(0-10 \mathrm{~nm})$
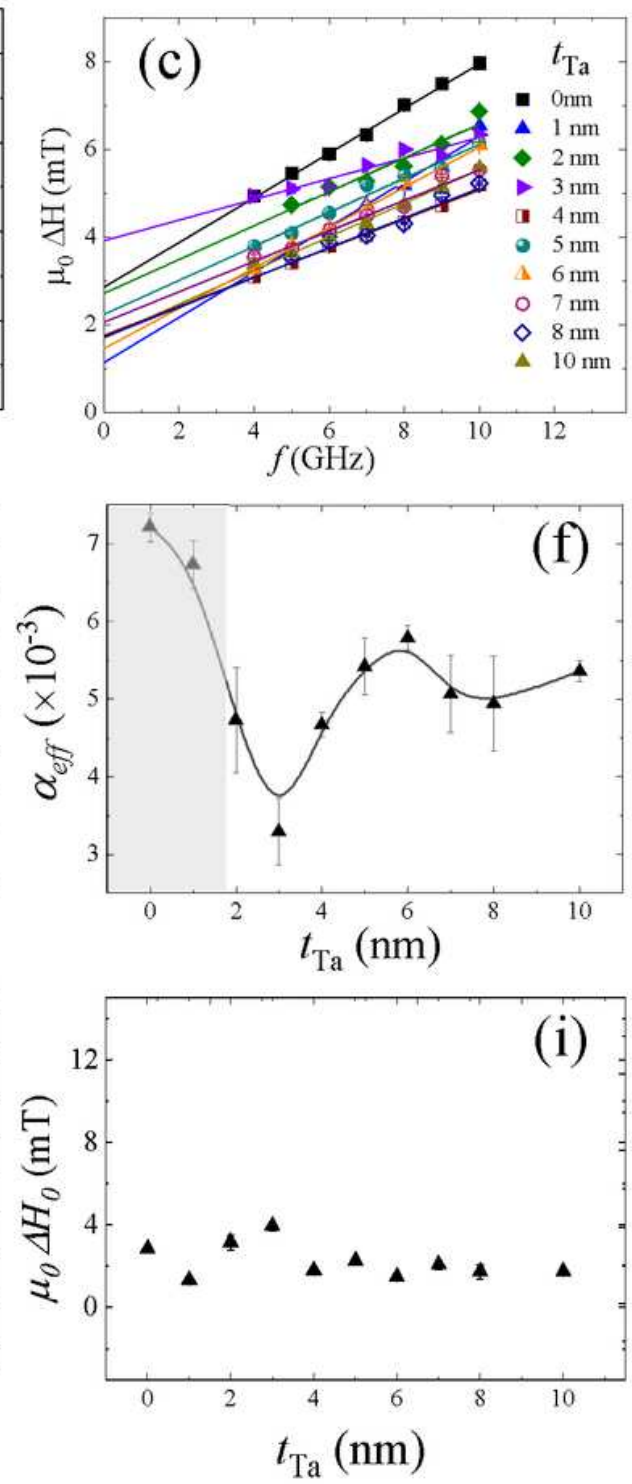

\section{Figure 9}

(a-c) Linewidth vs. frequency [lines are fitted to the experimental data using Eq. (4)]. (d-f) Effective damping constant vs tTa [Red line is fitted using Eq. 5.] (g-i) The inhomogeneous linewidth calculated using Eq. 3 by fitting of the data in Fig. 9 (a-c). Grey shaded region in Fig.9 (d, e and f) indicates the Ta gets affected with oxidation leads to the fluctuation in the damping constant. 